\title{
Arrival Time Distributions of Muons from Extensive Air Showers as Signature of the Mass Composition of Cosmic Rays
}

H. Rebel, G. Völker, M. Föller, A.A. Chilingarian Institut für Kernphysik 

KERNFORSCHUNGSZENTRUM KARLSRUHE

Institut für Kernphysik

KfK 5398

Arrival Time Distributions of Muons from $\mathbb{E x t e n s i v e ~ A i r ~}$ Showers as Signature of the Mass Composition of Cosmic Rays

H. Rebel, G. Völker, M. Föller and A.A. Chilingarian*

*Permanent address: Yerevan Physics Institute, Yerevan, Armenia

KERNFORSCHUNGSZENTRUM KARLSRUHE GMBH, KARLSRUHE 
Als Manuskript gedruckt

Für diesen Bericht behalten wir uns alle Rechte vor

Kernforschungszentrum Karlsruhe $\mathrm{GmbH}$ Postfach 3640, 76021 Karlsruhe

ISSN 0303-4003 


\section{Abstract}

The central detector of the KASCADE experiment which intends to study the main components of extensive air showers will be equipped with a trigger and fast timing facility which enables measurements of the arrival time distributions of the muons. In particular, at larger distances from the shower axis the arrival time distributions are expected to map the longitudinal development of the showers in the atmosphere and may provide additional information about the nature of the primary particle through differences of the mean-free paths and the interaction cross sections with air nuclei.

We have investigated this aspect with respect to the KASCADE setup on the basis of EAS-simulations using the Monte-Carlo code CORSIKA. The mean arrival time distributions of muons, observed at various distances from the shower core and for different primary energies and showers sizes, respectively, have been analysed with advanced statistical techniques, based on Bayes decision rules and nonparametric multivariate analysing methods, in order to specify the merits of the mean arrival time information of the muon component as a signature for the mass composition of cosmic rays. It turns out that muon arrival time distributions, observed with the KASCADE facility, exhibit promising features and mass discrimination effects for larger shower sizes, i.e. higher primary energies, around $10^{16} \mathrm{eV}$. 


\section{Ankunftszeitverteilungen von Myonen aus ausgedehnten Luftschauern als Signatur der Massenzusammensetzung der kosmischen Strahlung}

Der Zentraldetektor des KASCADE Experiments, mit dem die Hauptkomponenten der großen Luftschauer bestimmt werden sollen, ist mit einem Trigger und schnellen Zeitdetektor ausgerüstet, die die Messung von Ankunftszeitverteilungen von Myonen ermöglichen. Insbesondere in größeren Abständen von der Schauer-Achse liefern die Ankunftszeitverteilungen von Myonen Informationen über die longitudinale Entwicklung der Schauer und die Natur der Primärteilchen.

Wir haben diesen Aspekt für den KASCADE-Aufbau mit Hilfe von Monte-Carlo-Simulationen mit dem Code CORSIKA untersucht. Die "beobachteten" Zeitverteilungen und ihre Abhängigkeit von der Primärenergie bzw. Schauergröße sowie vom Abstand zum Schauerzentrum wurden mit Hilfe fortgeschrittener Methoden der statistischen Analyse studiert, um den Charakter der Informationen aus Ankunftszeitverteilungen über die Masse des Primärteilchens zu spezifizieren. Es stellt sich heraus, daß die Myon-Ankunftszeitverteilungen, die mit der KASCADE-Anlage beobachtet werden können, vielversprechende Effekte für die Massendiskriminierung zeigen, insbesondere für größere Schauer, d.h. höhere Primärenergien um $10^{16} \mathrm{eV}$. 


\section{Introduction}

High energy primary cosmic rays induce extensive air showers (EAS) in the Earth's atmosphere by cascading interactions with air nuclei. The produced secondary particles, reaching the ground level, carry information on the ultrahigh energy interaction and about the nature of the primaries.

In principle, the history of the cascade development of extensive air showers is reflected by the time structure of incidence. This is particularly true for the muonic component which conserves the geometrical structure of the air shower due to the reduced influence of multiple scattering and the higher penetrability of muons traversing through the atmosphere [1,2]. Thus, especially at larger distances from the shower axis where the geometric factors are dominating, the arrival time distributions map the longitudinal development of the shower in the atmosphere and may inform on early stages of the shower. Under this aspect the temporal characteristics of EAS muons has been first experimentally studied by Linsley and Scarsi [1], followed by various different attempts, in particular with the Haverah Park detector arrangements [3, 4] and other setups [5-7]. As the longitudinal development is determined by the multiplicity of the secondary particles from collisions of the leading particle and by the mean free path (and the cross sections of the interaction with air nuclei), the measurement of muon arrival time distributions and of the time dispersion of the muon front may provide signatures for the elemental composition of the primary cosmic rays. In the energy range around $1015 \mathrm{eV}$ the knowledge of the elemental composition is of great astrophysical importance and provides clues with respect of the questions about origin, acceleration and propagation of cosmic rays through interstellar space.

The muon arrival time distributions and the mean time dispersal, representing the thickness of the muon disc are dependent on primary energy, shower core distance, zenith angle of incidence and on the threshold energy of the muon detection. In order to get a quantitative insight into these features and to specify the experimental requirements of measurements, especially in context with the KASCADE experiment [8, 9], we have analysed data resulting from realistic Monte-Carlo simulations of extensive air showers, initiated by protons and Fe-nuclei in the energy range of $1014-1016 \mathrm{eV}$. The influence of various observation parameters on the distributions is studied, and correlations with various other observables are explored, using advanced statistical procedures and decision rules $[10,11]$. In order to approach the experimental situation of 
the actual detector setup of KASCADE, the response, the timing quality and trigger conditions of the experimental facilities are taken into account.

\section{Simulation of Extensive Air Showers}

The extensive air shower data have been generated by detailed Monte-Carlo calculations of the shower development in the atmosphere using the computer program CORSIKA [12]. This program has been specifically developed to perform simulations for the KASCADE experiment presently under construction at the site of the Karlsruhe research center. Generally, whereever possible, experimentally accessible data have been used as a basis for modelling the high-energy interactions of particles with air nuclei. All secondary particles are tracked explicitly along their trajectories, and their parameters are stored on tape when reaching an observation level. This allows a detailed analysis of all features of the simulated showers.

The CORSIKA code uses a Monte-Carlo generator based on the Dual Parton Model [13, 14]. This model describes the hadronic interactions of protons at high energies in agreement with the measured collider data. The model is extended to the interaction of primary nuclei by calculating the fraction of interacting nucleons in target and projectile, invoking the superposition hypothesis. Diffractive reactions are included. For the simulation of the electromagnetic component the EGS4 code [15] is included, but in most of our present calculations the NKG approximation is used. Details of the program are given in Ref. [12]. Actually, the present calculations use a modified and improved version of the CORSIKA program, differing by the treatment of the hadronic interaction below $\mathrm{E}_{\mathrm{cm}}=12 \mathrm{GeV}$ and replacing the previously used isobar-model description by an interaction model as worked out in the GEISHA-program [16].

For a data basis of the analysis a sufficiently large number of proton and iron induced air showers has been calculated, at nine values of the primary energy $E_{0}$, in equidistant steps on the $\log \mathbf{E}_{0}$-scale between 1014 and $1016 \mathrm{eV}$. Using the results a realistic shower size $\left(\mathrm{N}_{\mathrm{e}}\right)$ spectrum has been deduced, however ignoring the realistic mass composition and only considering the proton and iron component of primary cosmic rays. For sake of simplicity the analyses are restricted to showers of vertical incidence. 
Fig. 1 displays the longitudinal development of the electromagnetic and muonic components of air showers initiated by $1 \mathrm{PeV}$ protons and $1 \mathrm{PeV} \mathrm{Fe}$ nuclei, as calculated by the CORSIKA code.

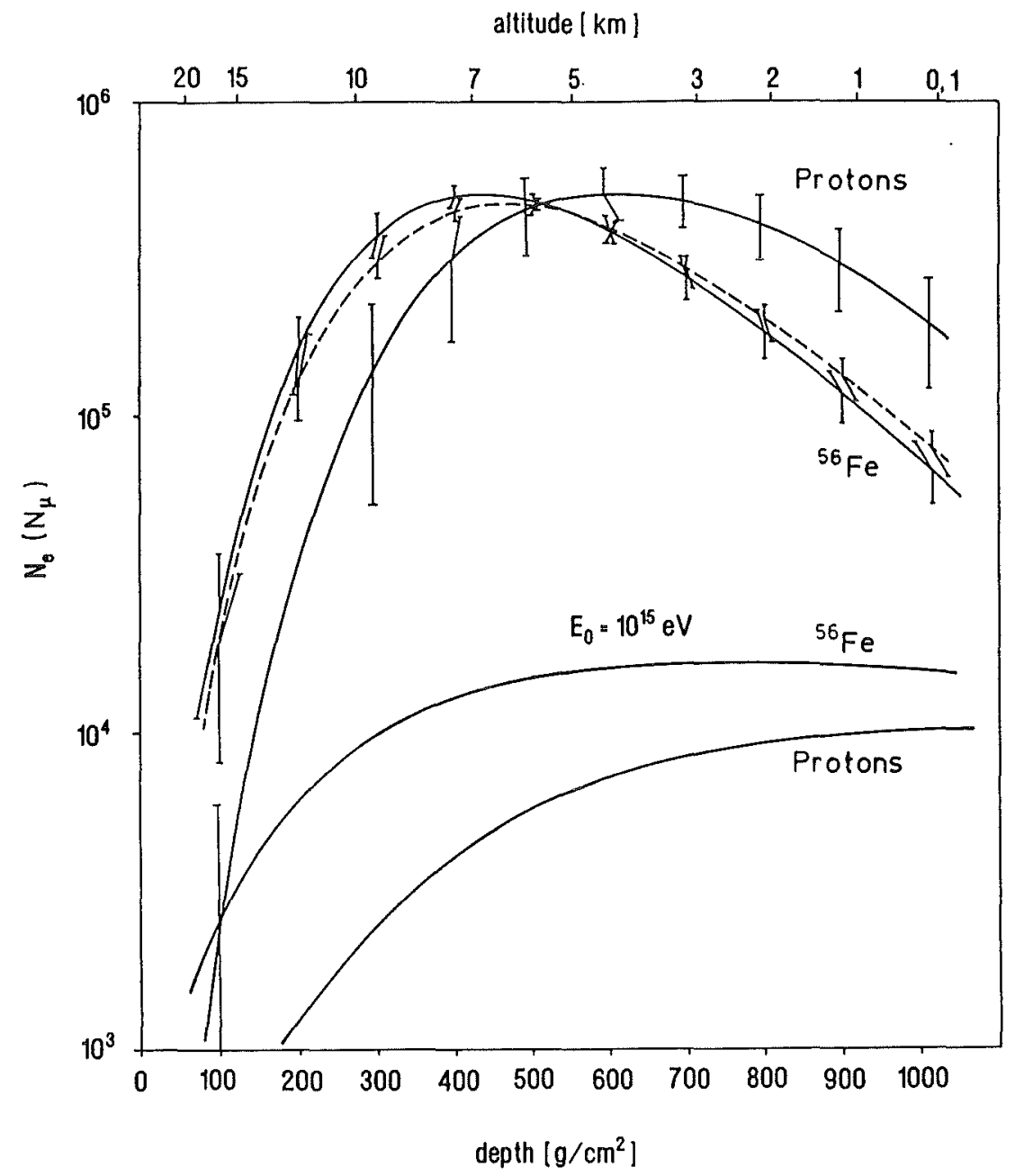

Fig. 1: $\quad$ Longitudinal development of the e/ $\mathrm{Y}$ and muonic components of $1 \mathrm{PeV}$ proton and $1 \mathrm{PeV}$ iron showers. The "error bars" at the $N_{e}$-curves represent the fluctuations, the dashed " $\mathrm{Fe}$ "curve indicates the effect of a different nuclear fragmentation pattern [17].

A powerful signature of primary mass is deduced from the muon multiplicityelectron size ratio, as observed at the observation level and corroborated by some additional information about the energy and lateral distributions of hadrons in the shower core, e.g. The faster development of the heavy primary induced shower, due to the smaller interaction length, is expected to be reflected by the mean arrival times of the muons. 


\section{Muon arrival time distributions}

The mean arrival times of the muon component, inferred for relatively small distances from the shower core (regime of Lorentz effects) prove to be rather insensitive to the nature of the projectile. Visible effects discriminating proton and iron primaries show up at larger core distances when path length effects of the travelling muons become dominant. However, there the low intensity of the muons hardly allows to study the arrival time distributions of single showers and we can concentrate our analysis to the distributions of the mean arrival times averaged over the singles distribution. The features which discriminate different primaries are more pronounced for larger threshold energies of the muon detection, while higher primary energies shift the effects to larger core distances.

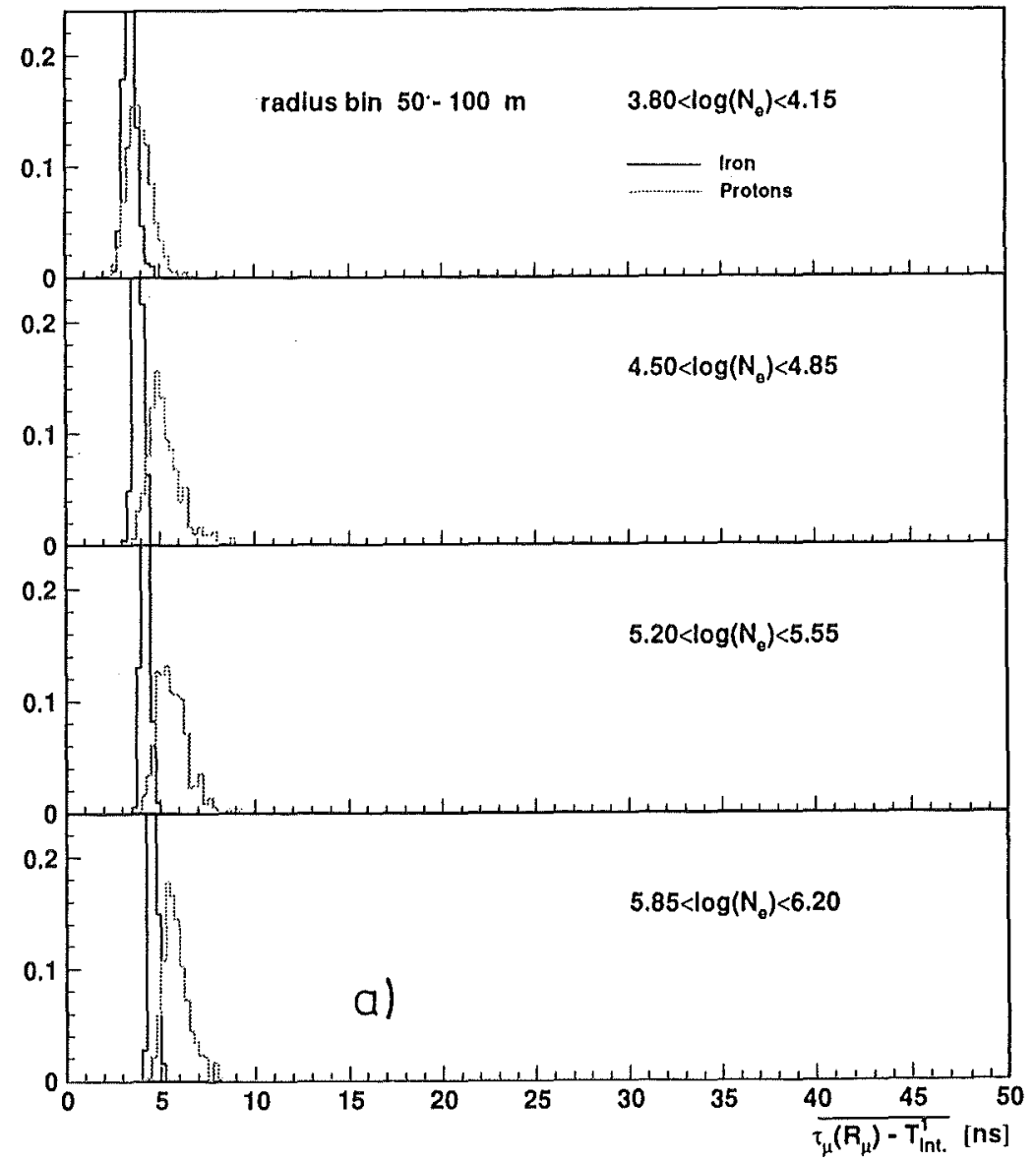

Fig. 2 a: Mean arrival time distributions for different ranges of the shower size and for a relatively small distance from the axis of proton and iron induced air showers, observed at sea level.The time-zero is the time $T^{1}{ }_{\text {int }}$ of the first interaction. 


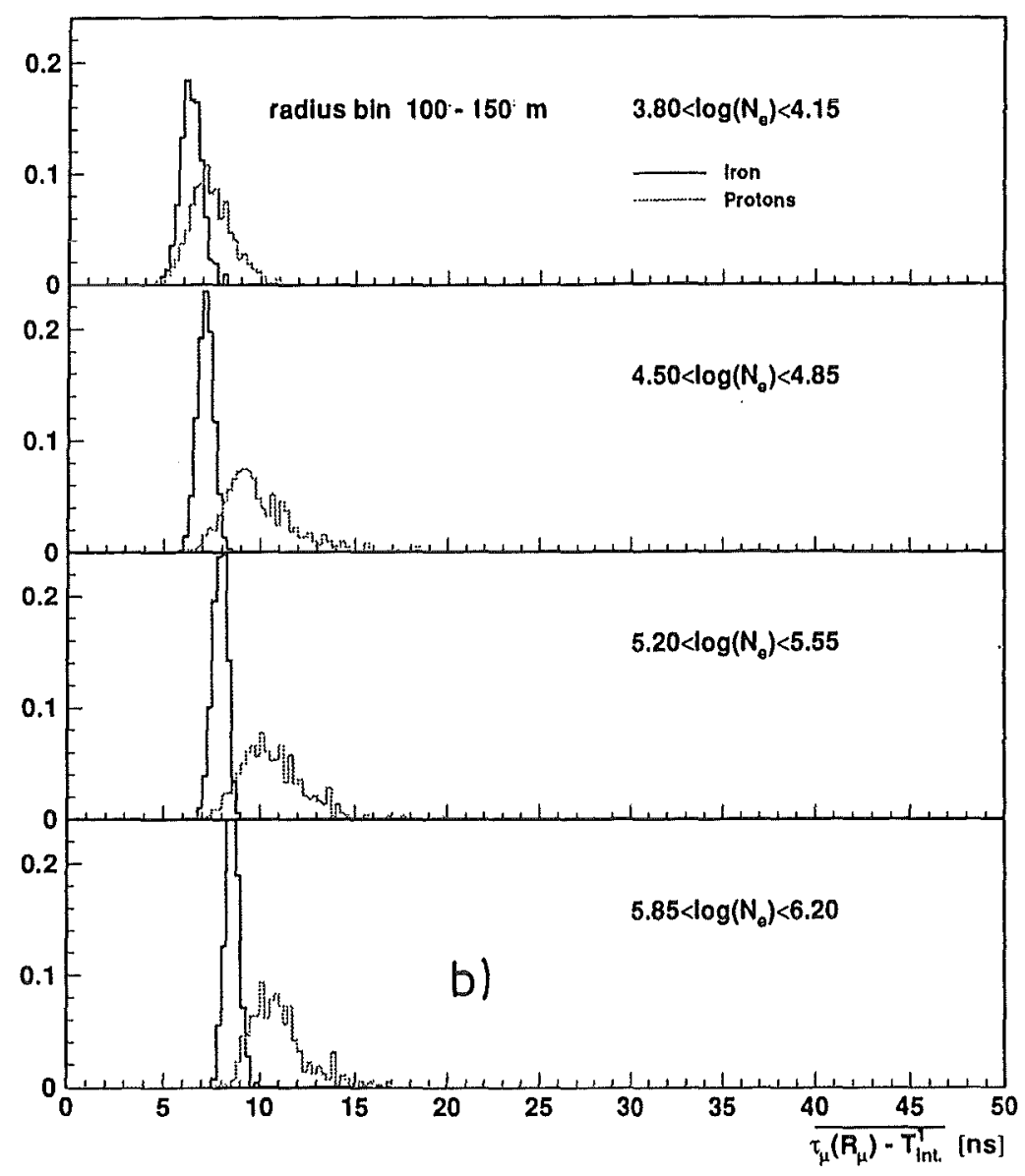

Figs. 2 b c:

Mean arrival time

distributions for

different ranges of

the shower size and

larger distances

from the axis of

proton and iron

induced air

showers, observed

at sea level.

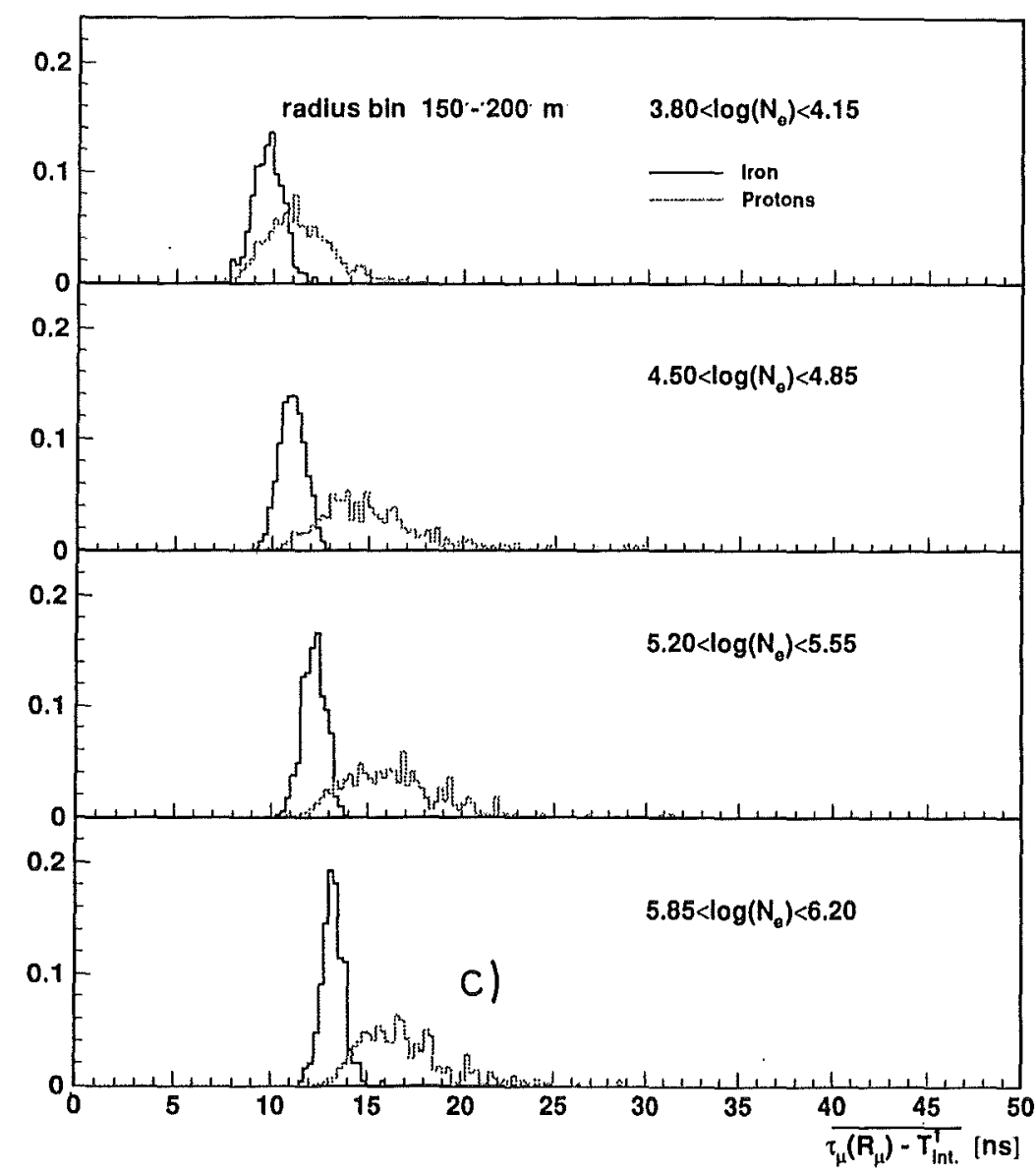




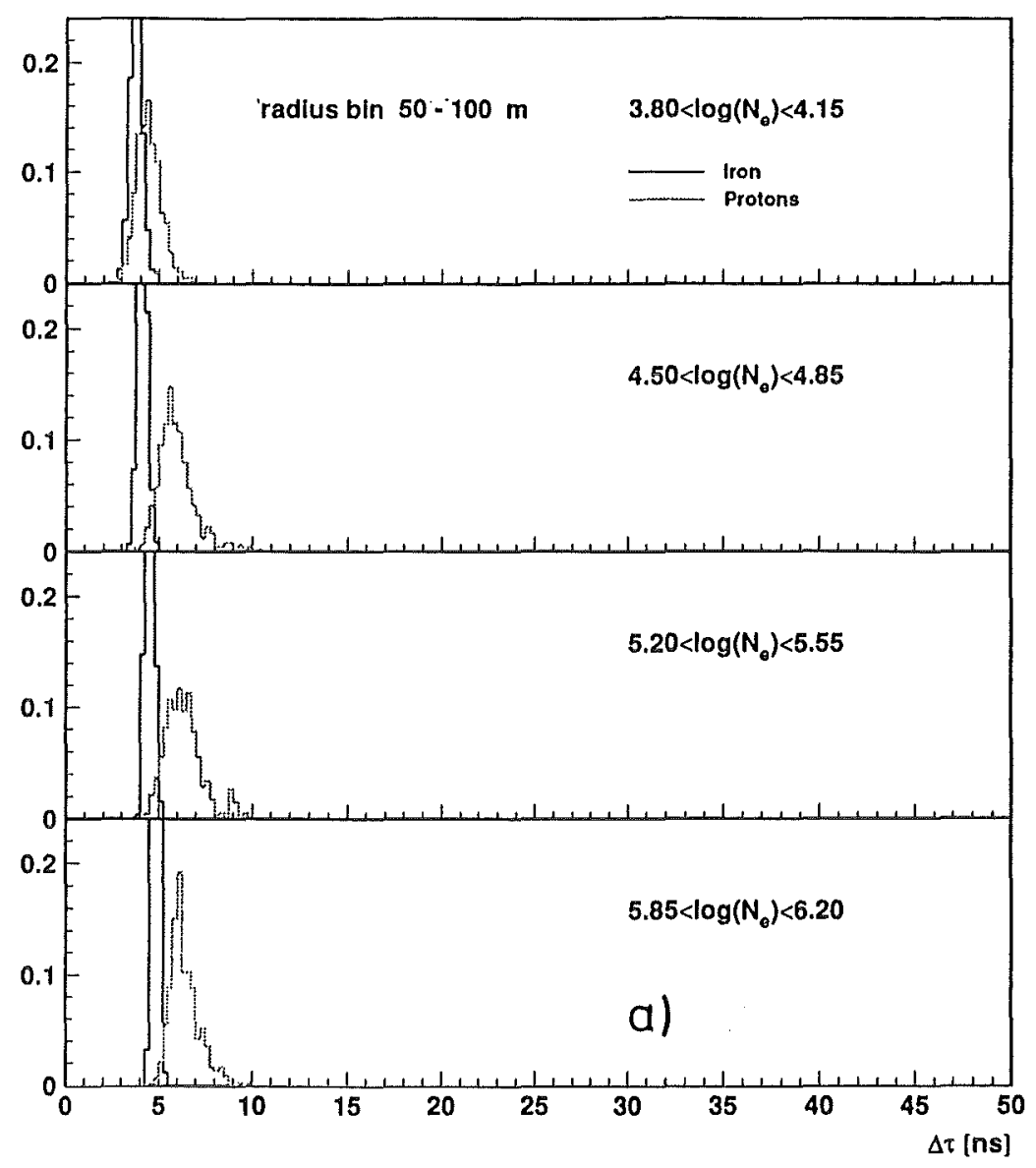

Fig. 3 a: Mean muon delay time distributions for different ranges of the shower size of proton and iron induced air showers.

Fig. $2 \mathrm{a} \mathrm{b} \mathrm{c}$ show mean arrival-time distributions of muons from the EAS simulations and with an energy threshold $\mathrm{E}_{\mathrm{th}}=2 \mathrm{GeV}$ (meeting the experimental situation, see sect. 4) in three radius bins of different distances from the shower axis, and for different ranges of the shower size. The calculated distributions have an offset in the time-scale, which is just the difference of the travel time of a light ray from the point of first interaction to ground level and the mean value of the arrival time of the muons of each shower. Thus the distributions reflect the fluctuation in the height of the first interaction and of the subsequent longitudinal development processes of the muon component.

The offset and the corresponding time-zero $\left(\mathrm{T}^{1}{ }_{\text {int }}\right)$ are not direct observables, and the distributions shown in Fig. 2 cannot be measured in this way. Experimental distributions take either the arrival of the first detected (local) muon as reference time $\left(\mathrm{T}_{\mu}{ }^{1}\right)$ which is expected to be a good representative of the foremost particle of the shower disc [6] (but additionally affected by considerable fluctuations with respect to $\mathrm{T}^{1}{ }_{\text {int }}$ ), or the arrival of particles of the shower front in the core $\left(\tau_{c}\right)$. The difference from the arrival time $\tau_{\mu}\left(R_{\mu}\right)$ of muons (at the distance $\left.R_{\mu}=\left(\left(X_{\mu}-X_{0}\right)^{2}+\left(Y_{\mu}-Y_{0}\right)^{2}\right)^{1 / 2}\right)$ 


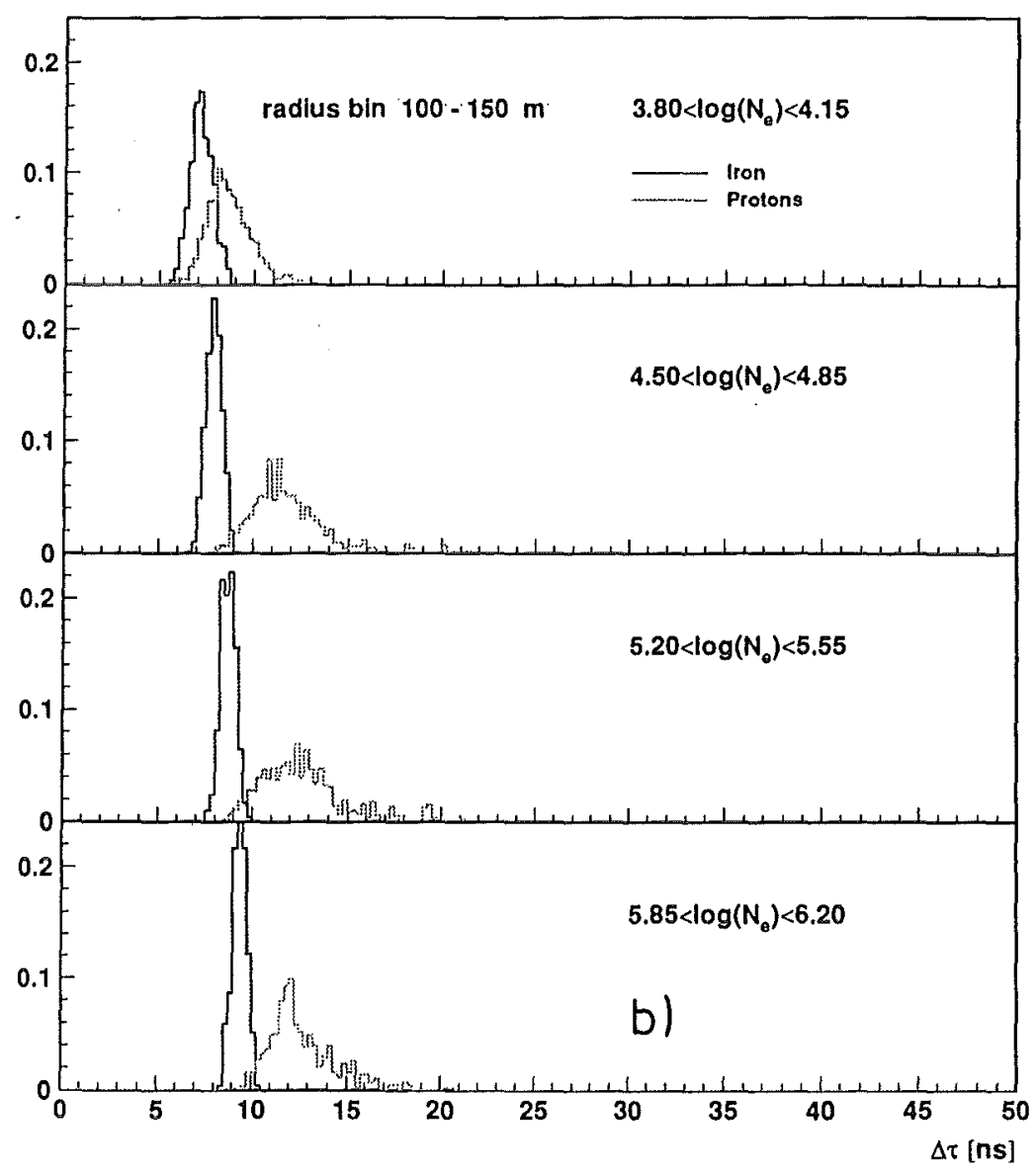

Figs. 3 bc:

Mean muon

delay time

distributions for

different ranges

of the shower size of proton and iron induced air showers.

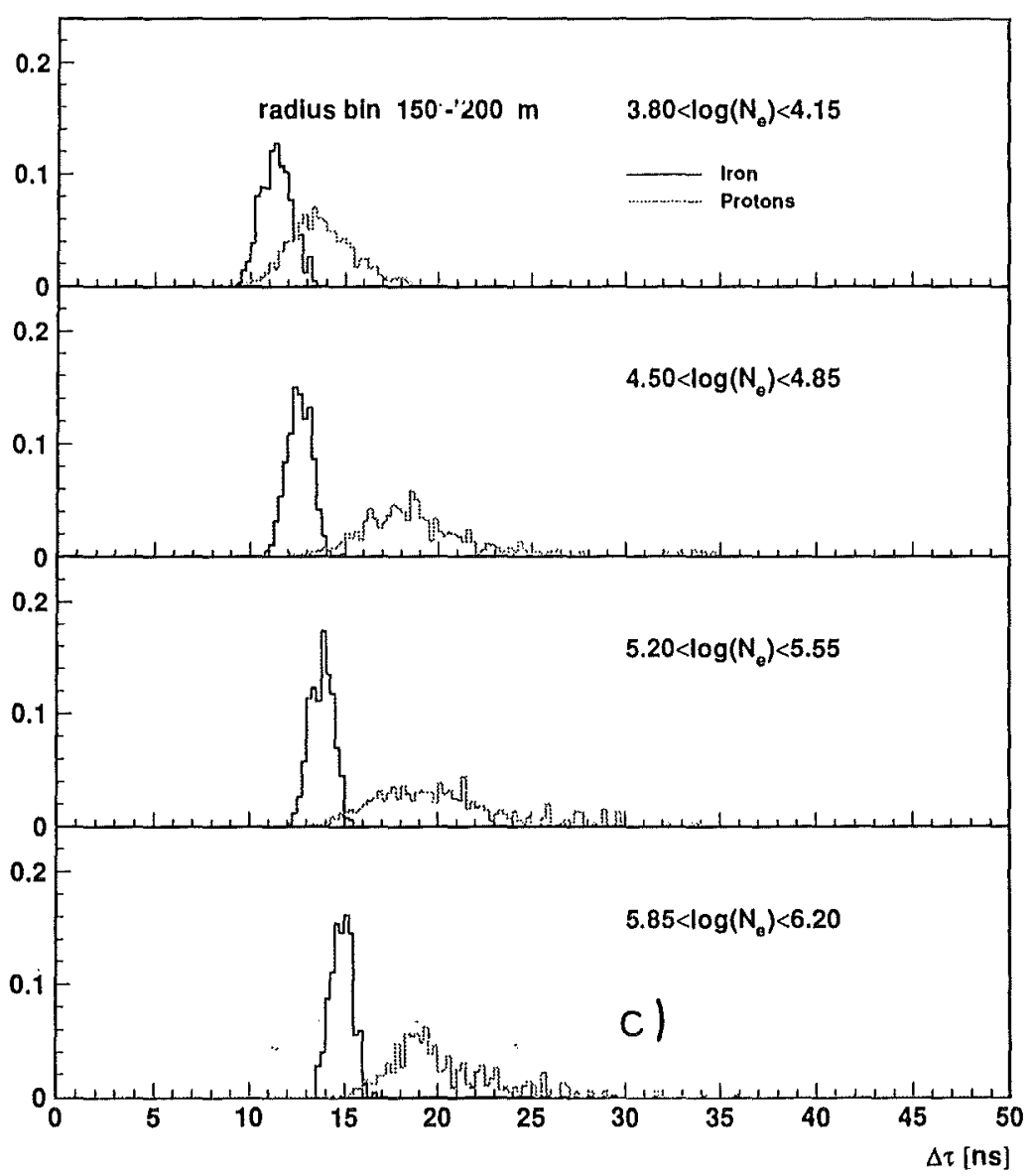




$$
\Delta \tau=\tau_{\mu}\left(R_{\mu}\right)-\tau_{c}
$$

is traditionally called the muon delay time. Mean muon delay time distributions (corresponding to the distributions shown in Fig. 2) are displayed in Fig. 3. Due to the different time-zero with different fluctuations these (observable) distributions appear to be slightly different. In the following, when additionally including the response and detector performance of the timing facility into the analysis of the time arrival distributions, we refer just to the mean muon delay time $\Delta \tau$.

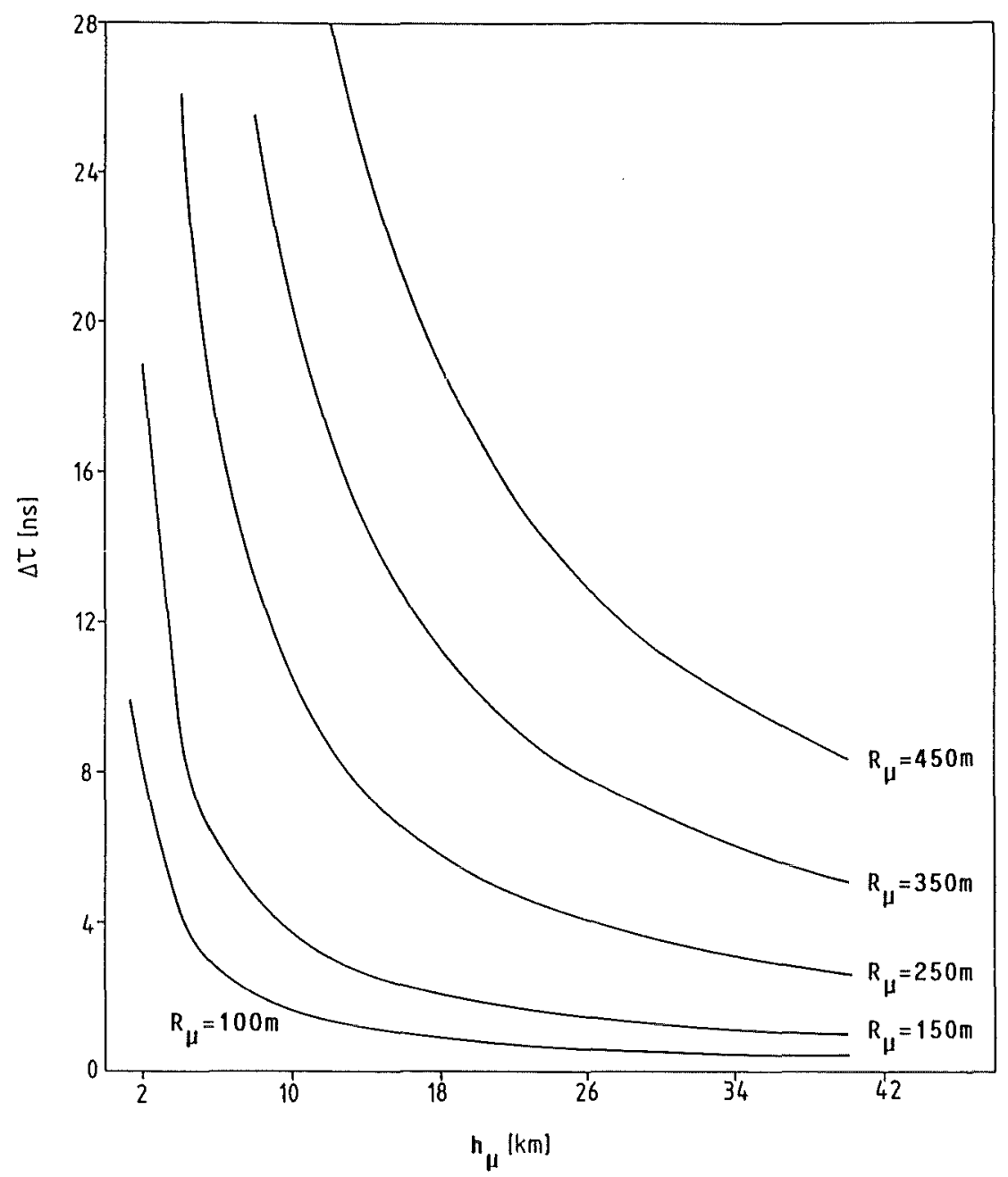

Fig. 4: Geometrical time delays of the muon arrrival time.

With respect to $\mathrm{T}^{1}{ }_{\text {int }}$ the muon arrival time is just the sum of the muon production time $\left(T_{0}\right)$ and the time of flight $\left(t_{\mu}\right)$ from the origin to the observation point. Introducing the travel distance $l_{\mu}$ of the muon from the origin and assuming that muons of higher energy are produced by the decays of hadrons flying with the shower front near the axis, we write 


$$
\begin{array}{ll}
t_{\mu}=l_{\mu} / \beta_{\mu} c & \left(\beta_{\mu}=\left(1-1 / Y^{2}\right)^{1 / 2}\right) \\
\tau_{c}-T_{o}=h_{\mu} / c &
\end{array}
$$

with $h_{\mu}=$ muon production height and $h_{\mu} / c$ the travel time of the electromagnetic front of vertical showers $\left(l_{\mu}^{2}=h_{\mu}{ }^{2}+R_{\mu}^{2}\right)$.

$$
\Delta \mathrm{\tau}=l_{\mu} / \beta_{\mu} c-h_{\mu} / c
$$

(neglecting effects of multiple scattering).

Thus, within a simple approach (which naively ignores all uncertainties arising from the real detector performance and details of muon propagation) the energy and the (mean) height of origin may be estimated. Additionally associating the muon delay completely to path length effects and assuming $\beta_{\mu}=1$ we arrive at:

$$
h_{\mu}=\frac{R_{\mu}{ }^{2}-(c \Delta \tau)^{2}}{2 c \Delta \tau}
$$

Eq. 3.4 (generalized for nonvertical showers [18] ) is the basis of the "timing method" for the determination of the muon production height, recently methodically combined with tracking studies ("Time-Track Complementary") and proposed for experiments by Danilova et al [18].

Fig. 4 displays calculated values of $\Delta \tau$ due to path length difference effects, which obviously dominate the features of the mean arrival time distributions exhibited by the Monte-Carlo simulations at larger $R_{\mu}$. It is obvious that large heights could only be studied at large $R_{\mu}$ and small delays. However there are additional effects due to the muon velocity dispersion leading to a time spread of muons produced in the same height but with different energies (see ref. 3 ).

In order to elucidate the origin of the muons arriving at observation level, Figs. $5 \mathrm{a}$ b display the arrival time distributions of the muons specified by the origin from different generations. Fig. 5a presents the mean arrival time distributions specified by the origin of the foremost muon which appears to be dominantly produced by the decay of the first secondary ( 2 nd generation). The distributions of the average generation of the events (Fig. 5b) show that most muons orginate from later generations consistently following the longitudinal development of the muon component (Fig. 1). 

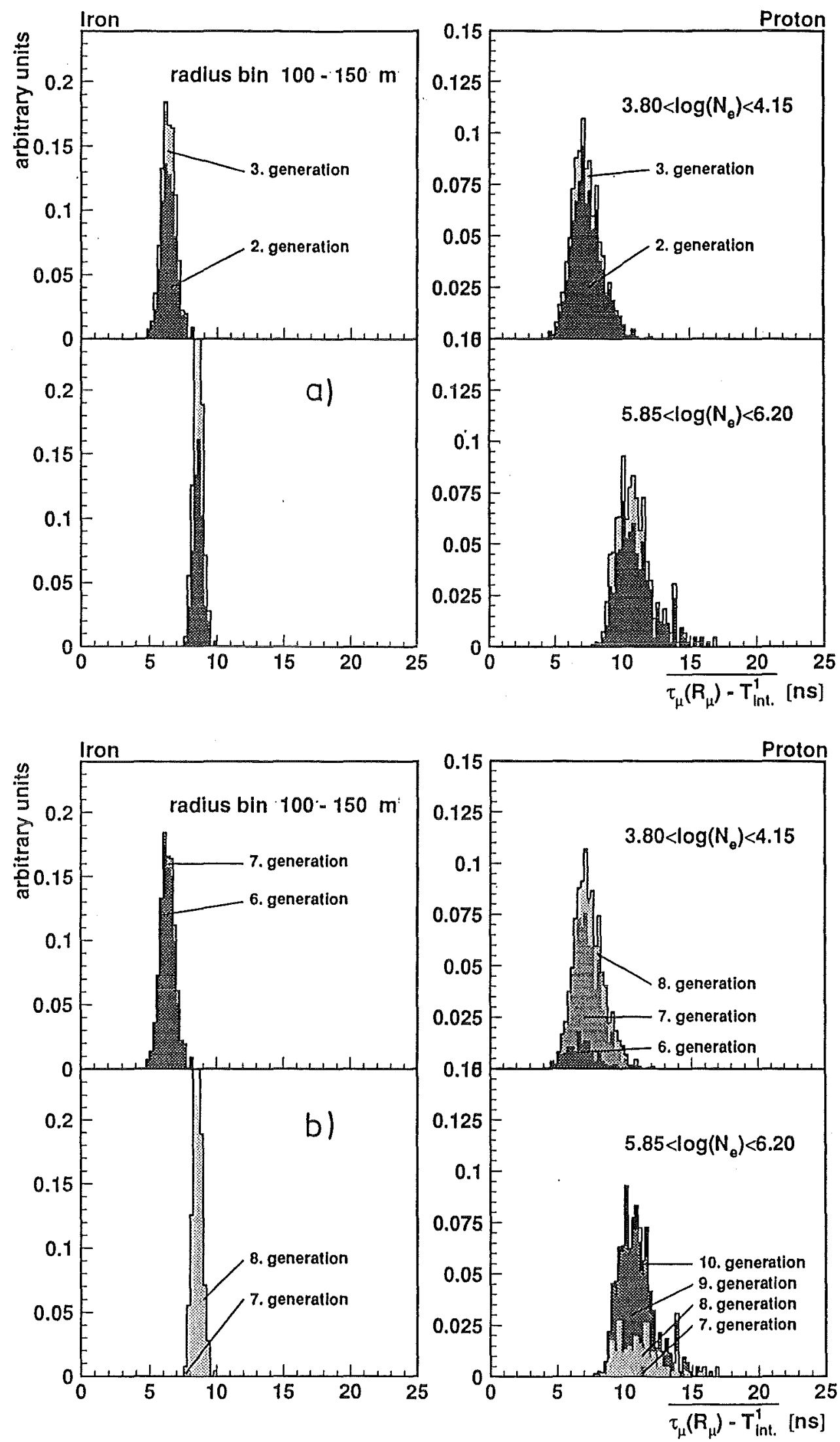

Fig. 5: Arrival time distributions decomposed by generation:

a. origin of the foremost arriving muon,

b. average generation of the arriving muon. 


\section{General layout of the KASCADE detector}

KASCADE which stands for "KArlsruhe Shower Core and Array DEtector" is an attempt to improve our experimental knowledge about the elemental composition of cosmic rays in an energy region where direct measurements by balloon-borne detectors e.g., are not feasible. The improvement is attempted $[8,9]$
- by simultaneous observation of many shower parameters of the electromagnetic, muon and hadron components,
- by a larger degree of sampling and
- by a correlation analysis of various signatures of the primary mass guided by Monte-Carlo simulations with an improved interaction model.

The KASCADE detector field comprises an array of detectors for the e/y and muon components with a total area of $200 \times 200 \mathrm{~m}^{2}$ and a central detector consisting of a hadron calorimeter of $20 \times 16 \mathrm{~m}^{2}$ (3800 $\mathrm{t}$ iron as passive layers) and $300 \mathrm{~m}^{2}$ multiwire proportional chambers (MPWC) in the basement below for muon detection. The array consists of 252 detector stations, organized in 16 subarrays, each forming a quadratic grid of $13 \times 13 \mathrm{~m}^{2}$ and will measure the electromagnetic and muonic components with their lateral distributions, thus providing the basic information about the shower size $\mathrm{N}_{\mathrm{e}}$, the location of the shower core $\left(\mathrm{X}_{0}, \mathrm{Y}_{\mathrm{o}}\right)$, arrival direction $(\theta, \phi)$, and especially of the arrival time $\tau_{c}$ of the shower front.

The central detector has various parts (Fig. 6):

- a sampling calorimeter $\left(20 \times 16 \mathrm{~m}^{2}\right)$ for measuring the energy spectrum and the lateral distribution of hadrons in the shower core

- multiwire proportional chambers installed in the basement for measurements of muons of an energy threshold of $2 \mathrm{GeV}$

- one active layer of the calorimeter setup is a trigger and timing facility, enabling arrival time measurements (i.e. of the thickness of the arriving shower disc)

- on top of the central calorimeter there is a special e/Y mini array : Top cluster (coupled to the full array) for studying special showers events (say, mini showers or muon-bundles without any $\mathrm{e} / \mathrm{Y}$ component) and the density in the core.

The detector field is covered with detectors to $2.5 \%$ for muons, $2,0 \%$ for electrons-photons and $0.6 \%$ for hadrons. The expected rates for showers with the core inside the array are $2 \mathrm{~s}^{-1}$ for $\mathrm{E}_{0}>10^{14} \mathrm{eV}$ and $20 \mathrm{~h}^{-1}$ for $\mathrm{E}_{0}>1016 \mathrm{eV}$. 


\section{KASCADE CENTRAL DETECTOR}

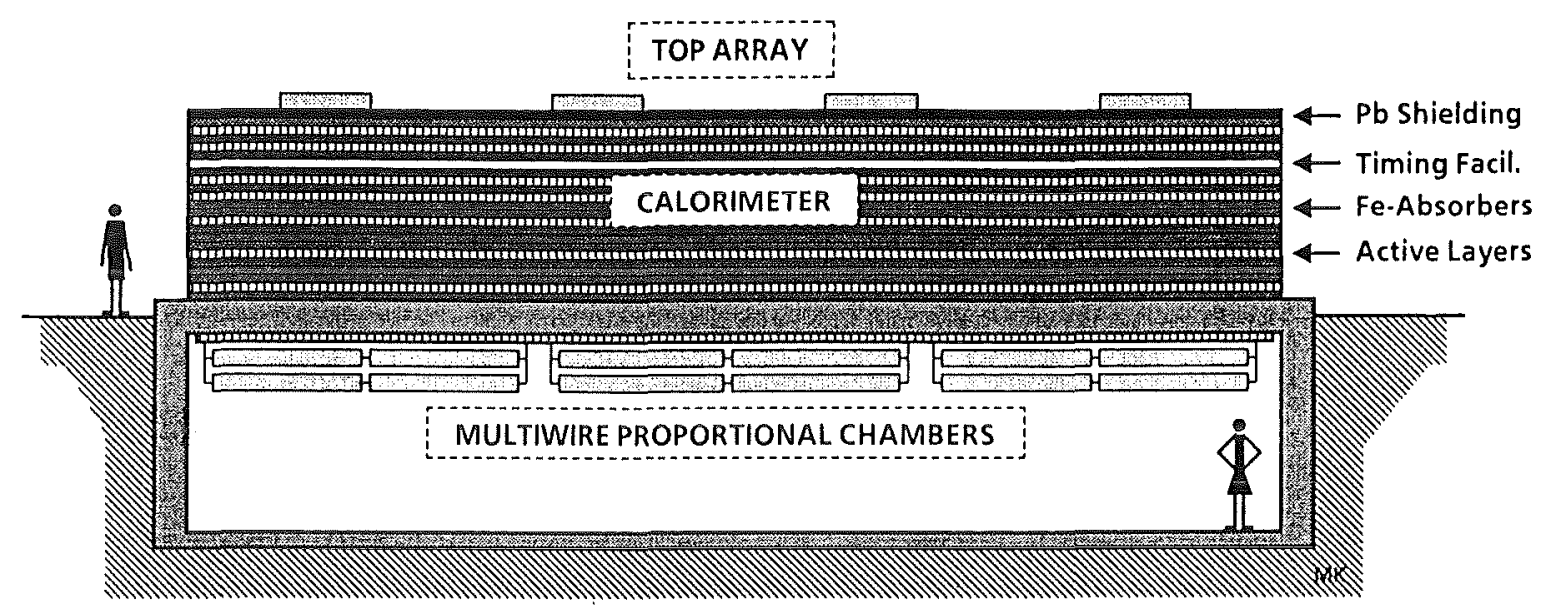

Fig. 6: Schematic view of central detector of KASCADE.

The active layers of the calorimeter are room-temperature liquid ionisation chambers [8].
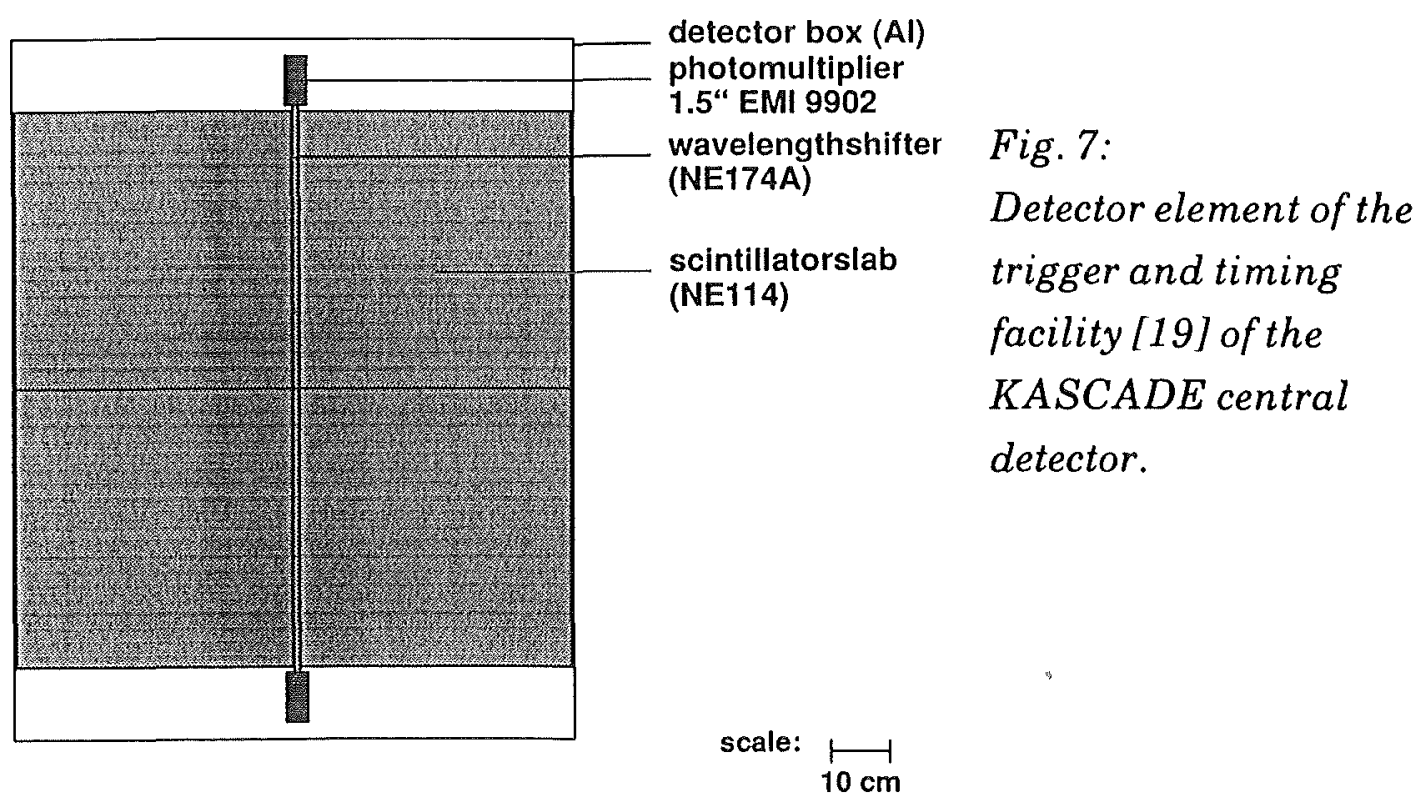

With respect to muon arrival time distribution measurements the most important parts of the KASCADE central detector are the fast trigger and timing detector in the third active layer of the calorimeter operated in coincidence with the muon identification and detection below the iron absorbers. 
This facility [19], primarily for triggering the active detectors in the calorimeter and the MWPCs, is built up by 456 detector elements covering $64 \%$ of the total central detector area. Each single element consists of two quadratic scintillator sheets of $3 \mathrm{~cm}$ thickness (Fig. 7), with an readout by a wavelength shifter bar and a 1.5" photomultiplier. Two of the detectors are housed, optically separated, in a detector box. The time resolution of the trigger detectors is $\sigma_{\mathrm{t}}=1.8 \mathrm{~ns}$.

\section{Different kinds of quasi-experimental muon arrival time distributions}

In the analysis including the experimental conditions of the KASCADE timing facility an acceptable event is defined by a coincidence multiplicity of at least 5 detectors units (supported by muon identification of the MWPC).

We call local muon delay time distribution the time delays of all detector events registered with respect to the first firing unit. The distributions of their mean values, including the effect of the detector geometry and of the response under the prechosen triggering conditions are displayed in Fig. 8 and demonstrate visibly the deterioration of the mass separation, due to the finite size of the timing detector setup together with considerable fluctuations of the local time-zero $\tau_{\mu}{ }^{1}\left(R_{\mu}\right)$ with respect to the time of the first interaction $T^{1}{ }_{\text {int }}$.

The effect of the finite detector size and of the low muon density at larger distances $R_{\mu}$ is directly evident when comparing the mean muon delay time distributions given in Fig. 3 with the corresponding "experimental" distributions of Fig. 9.

These "experimental" distributions do not include a time jitter and a relative time shift arising from the experimental determination of the arrival of the shower front $\left(\tau_{c}\right)$.

A relatively clear signal (Fig. 10) is provided by the muon delay

$$
\Delta \varepsilon_{\mu}^{1}\left(R_{\mu}\right)=\mathrm{c}_{\mu}^{1}\left(R_{\mu}\right)-\mathrm{c}_{c}
$$

of the foremost muon (with a large probability of originating from an early stage of the shower development). This is displayed by the distribution of $\Delta \tau^{1}$ Det ( $=\Delta \tau^{1} \mu$ with the detector efficiency taken into account). 
Mean arrival time including detector geometry

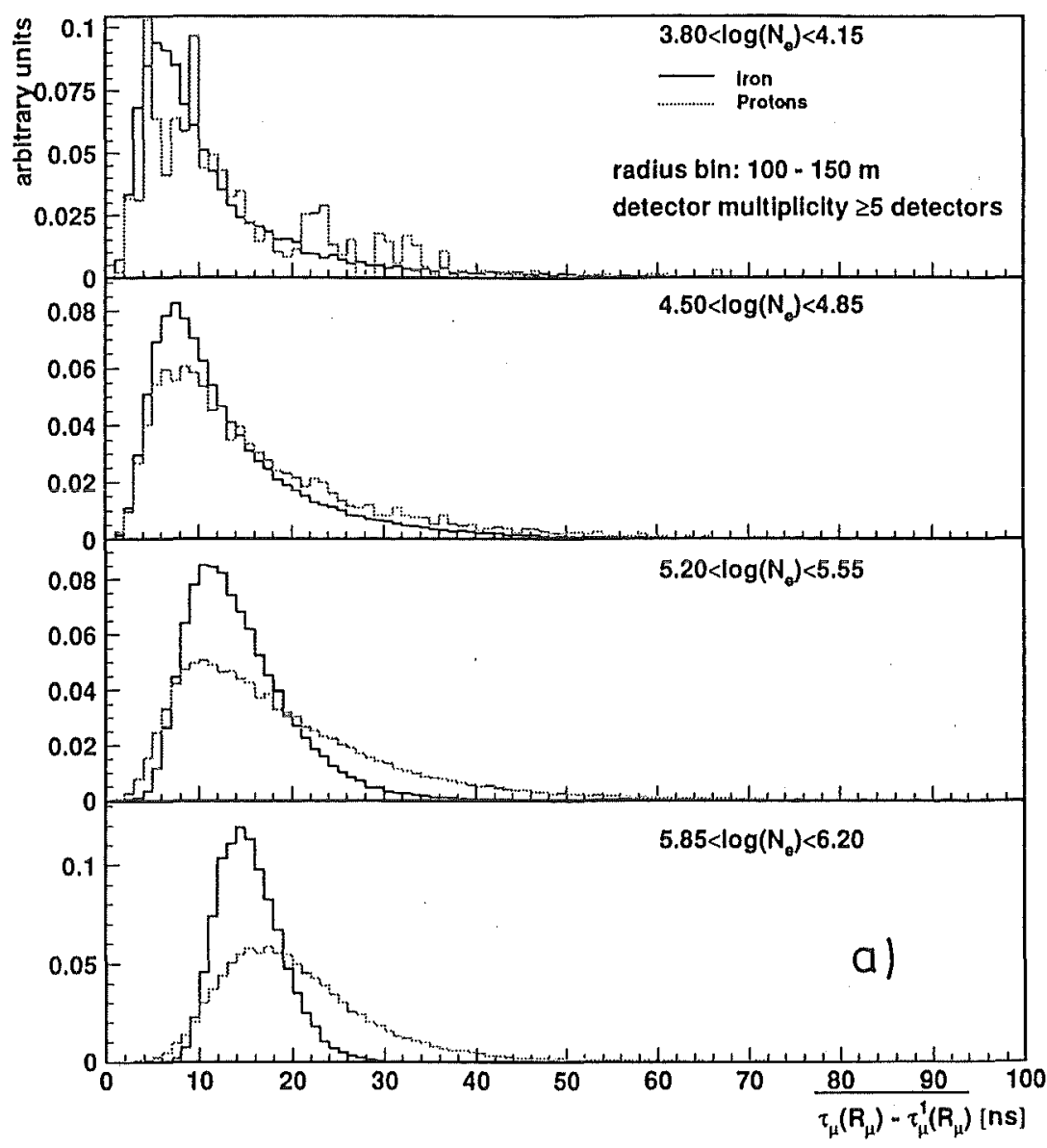

Fig. 8:

"Local" mean

arrival time

distributions

including

detector geo-

metry, response

and time-reso-

lution for the

muon multi-

plicity $\geqq 5$.

Mean arrival time including detector geometry

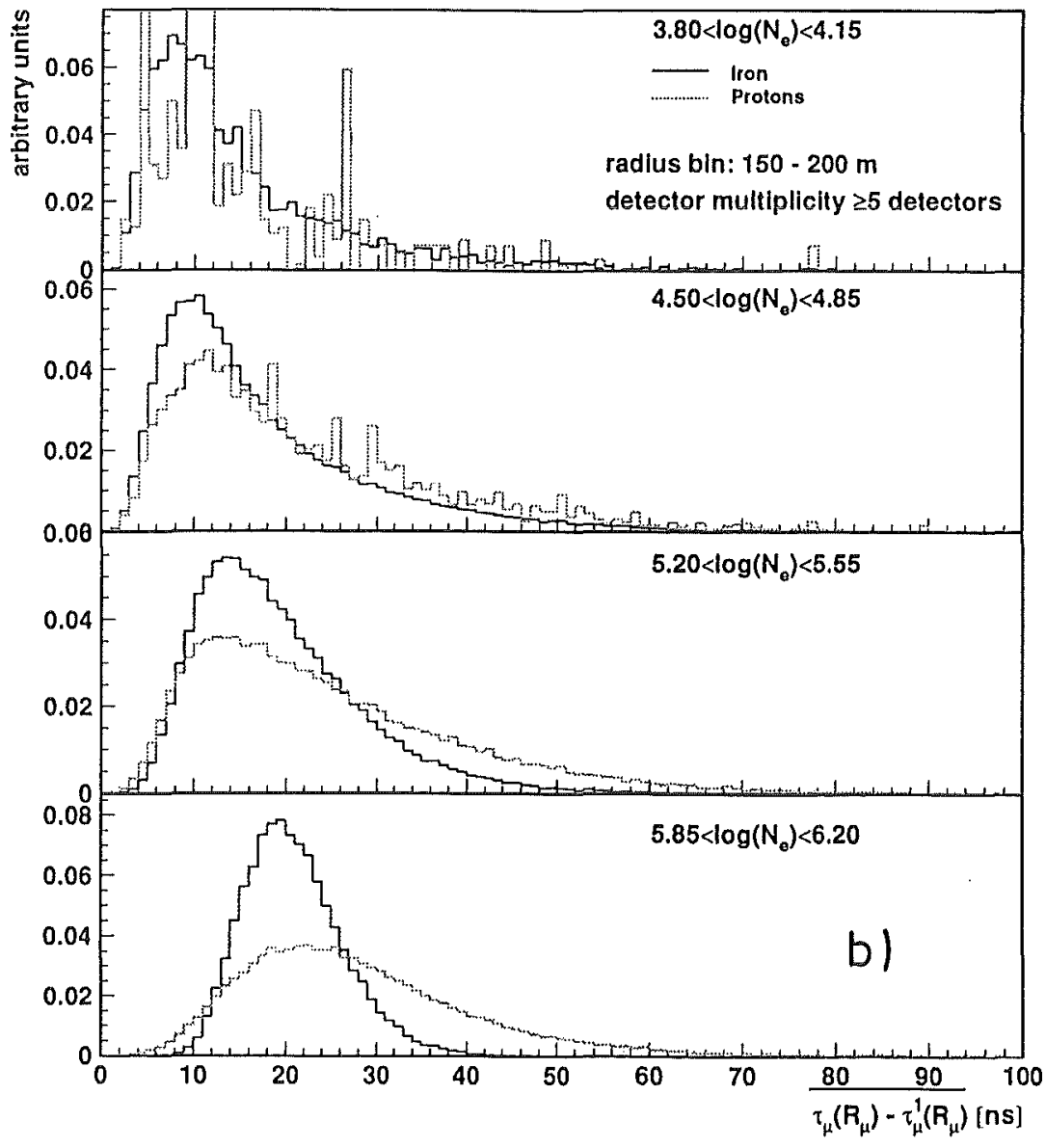




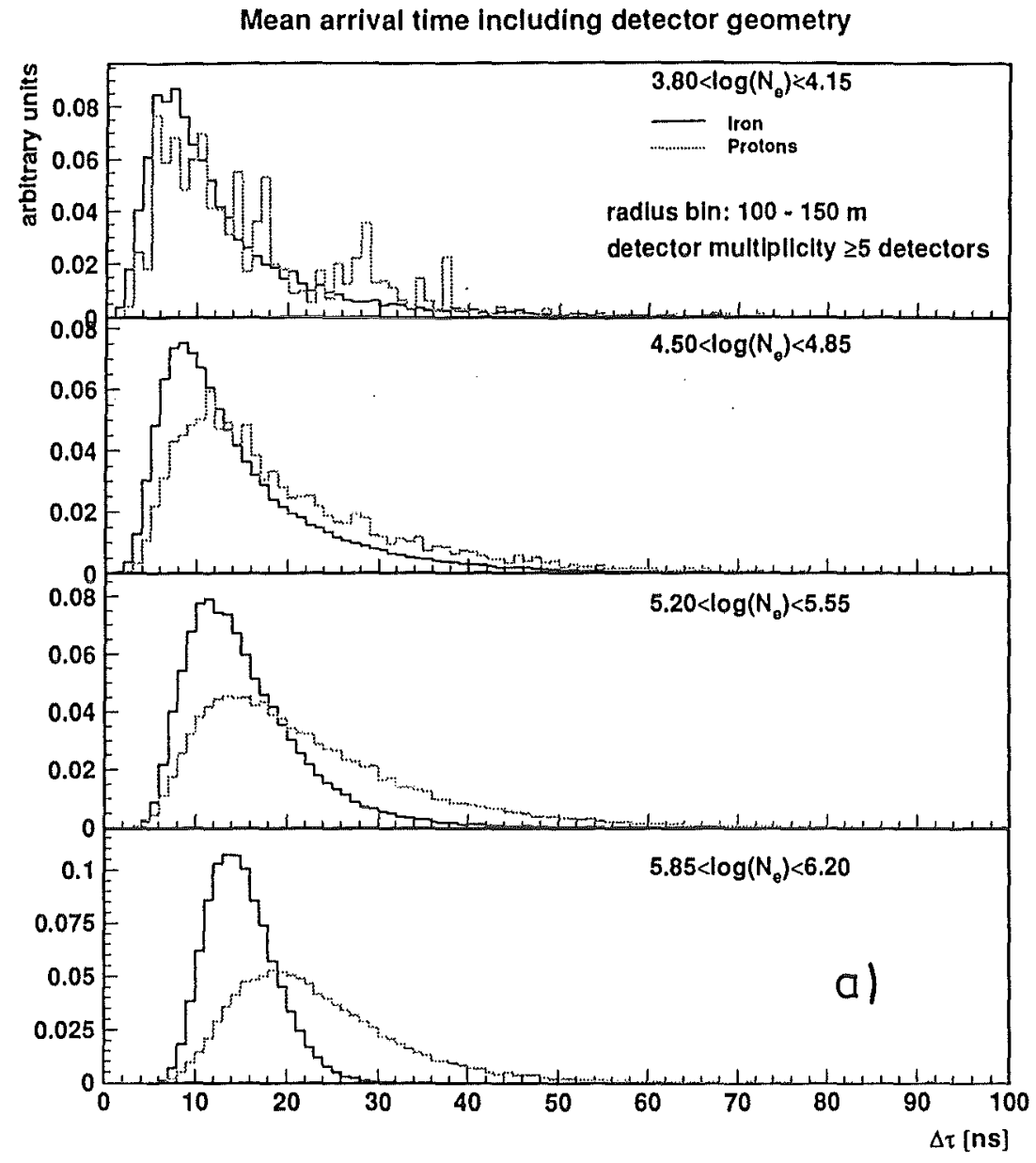

Fig. 9:

Mean muon

delay time

distributions

including

detector geo-

metry, response

and time-reso-

lution for the muon multi-

plicity $\geqq 5$.

Mean arrival time including defector geometry

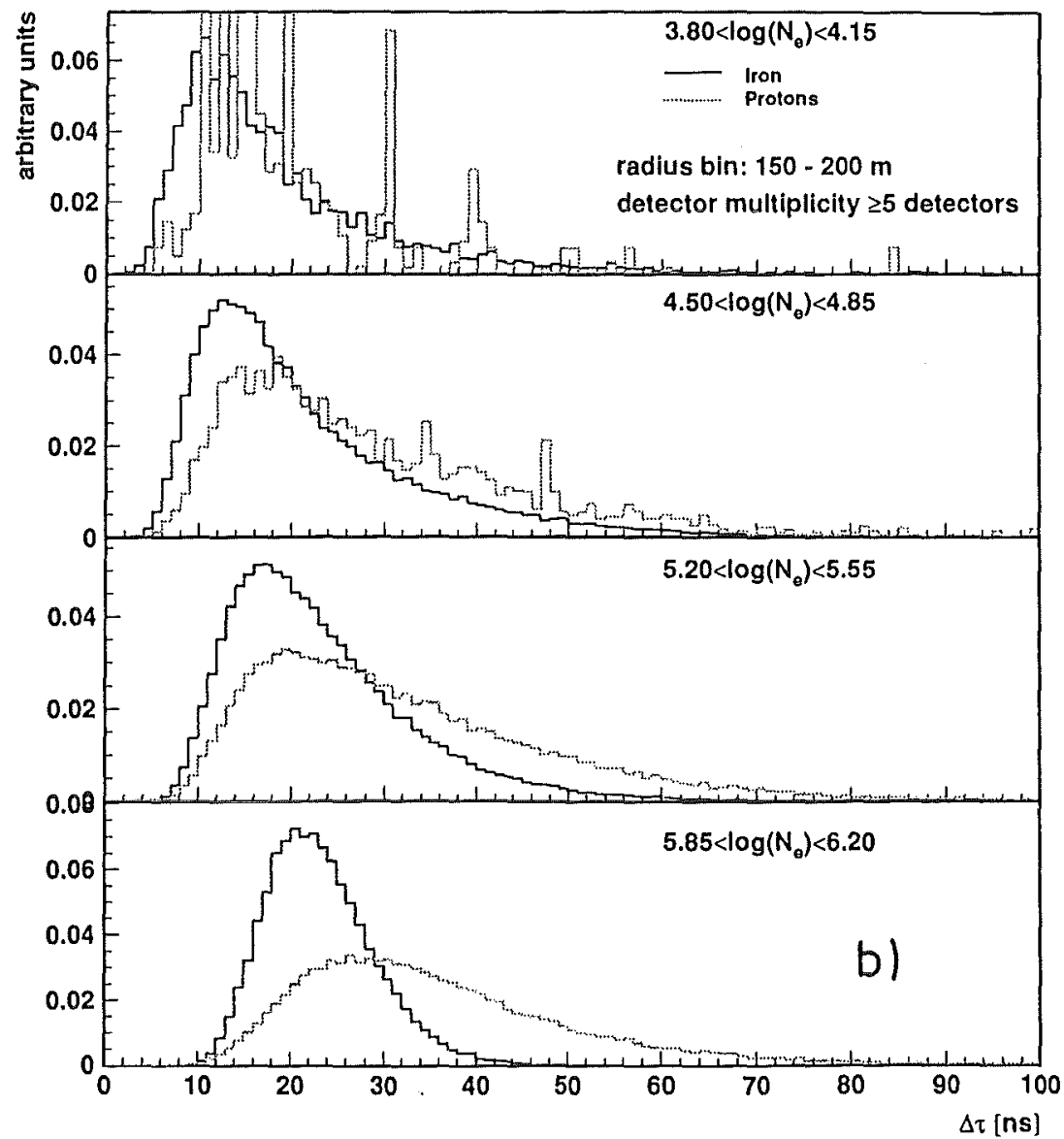


arrival time of first detector including detector geometry

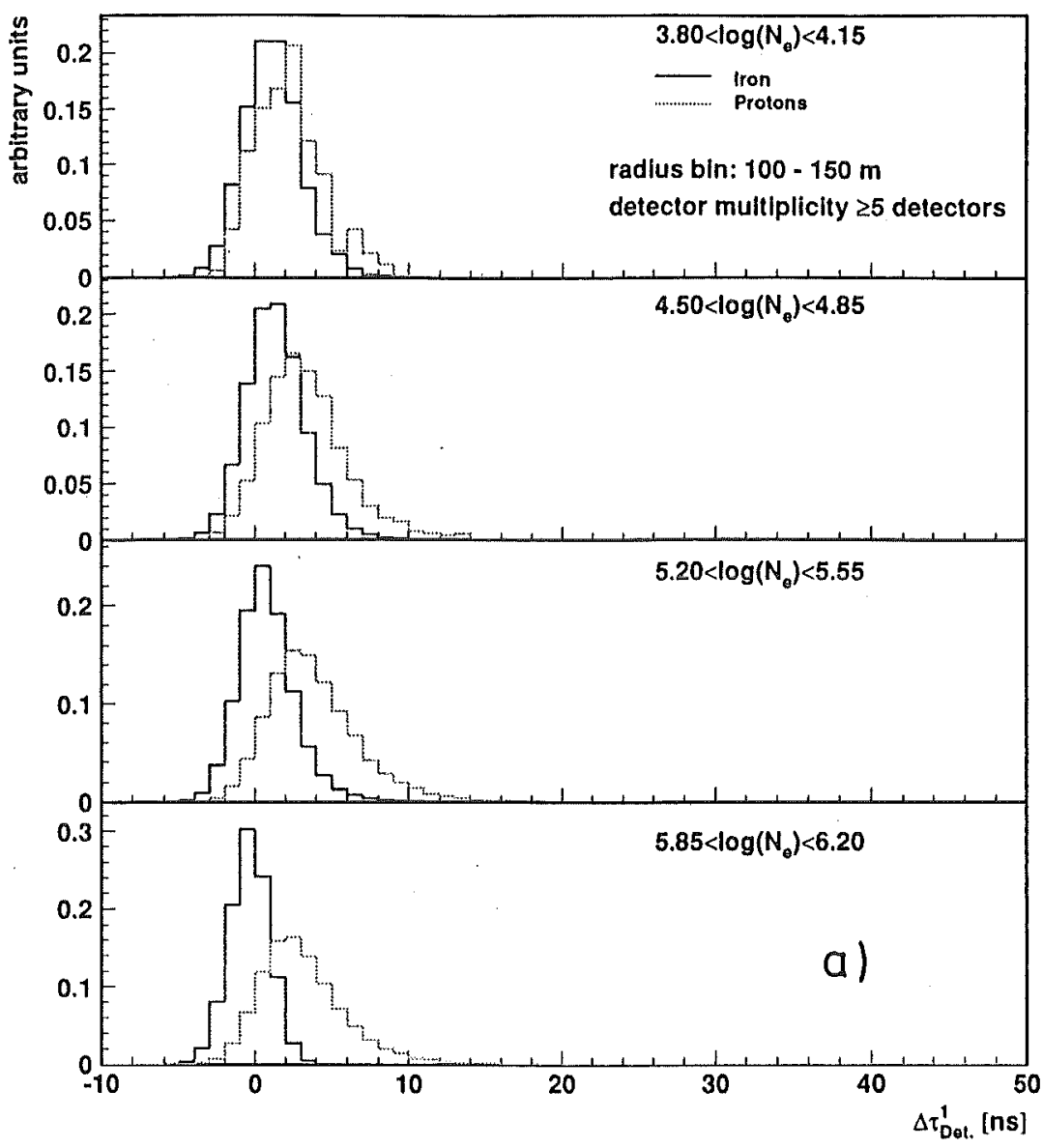

Fig. 10:

Delay time of the

foremost muon

(for a muon

multiplicity $\geqq 5$ )

in two different

$R_{\mu}$-bins and for

different ranges

of the shower

size (detector

efficiency taken

into account).

arrival time of first detector including detector geometry

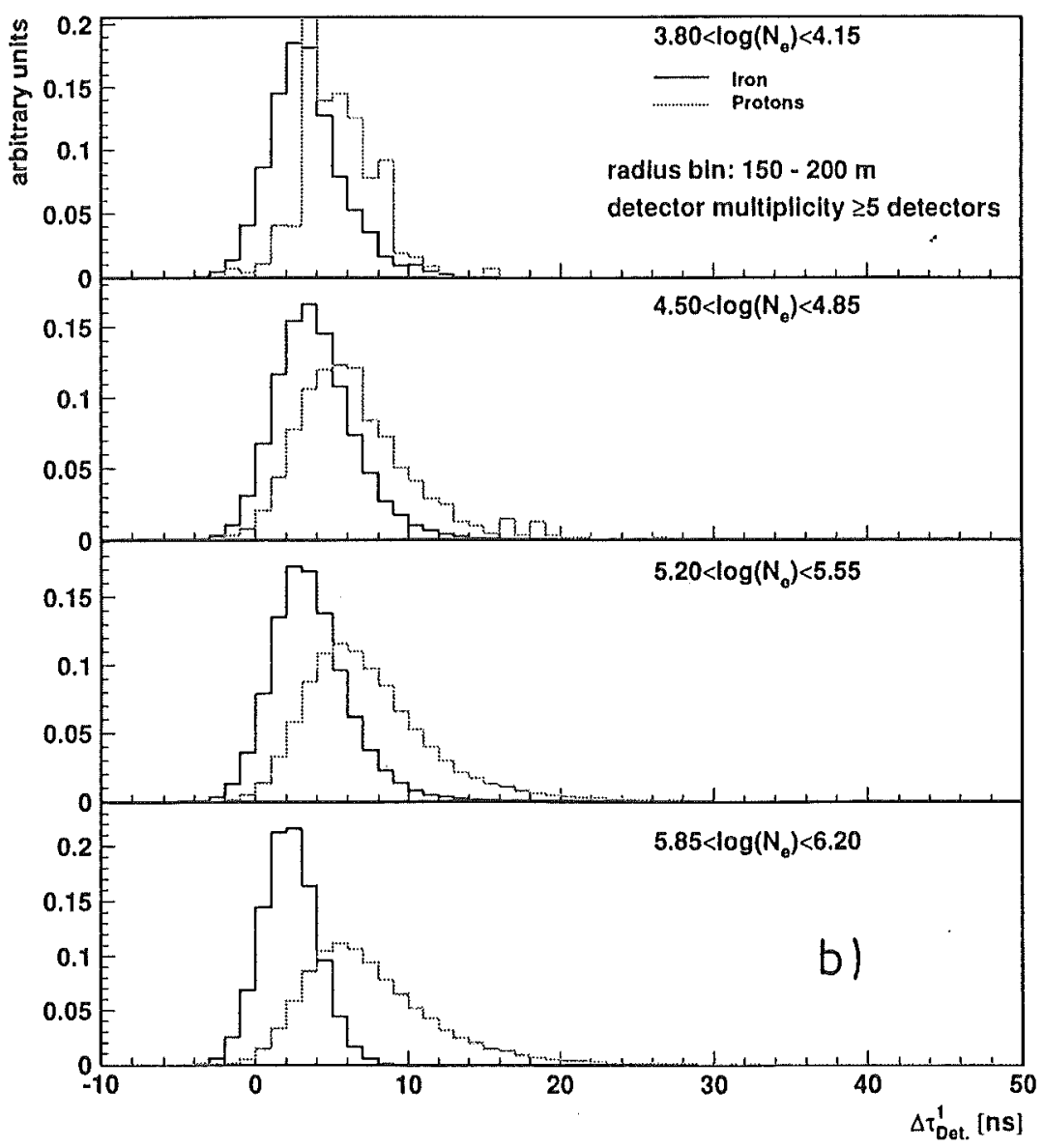



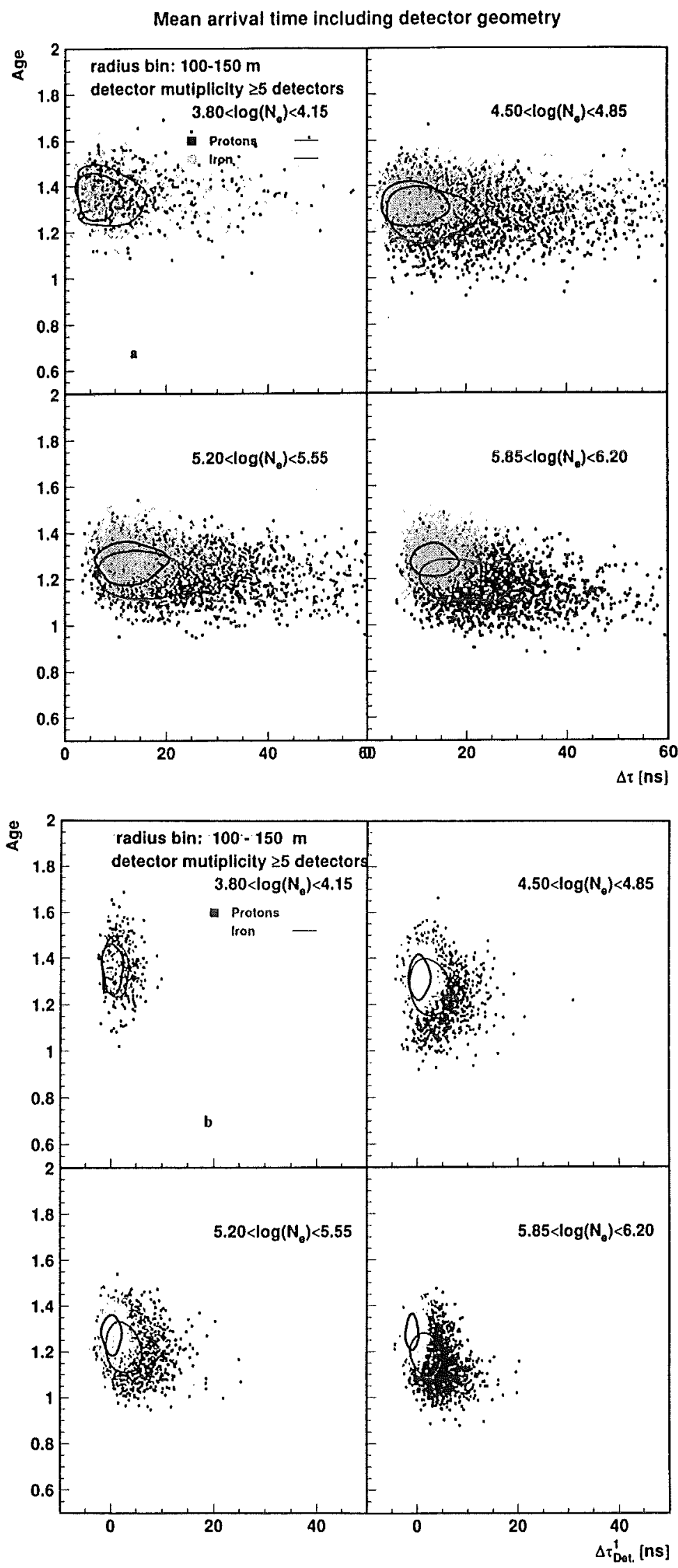

Fig. 11: Correlation of muon arrival times with the shower age $s$ a. $s-\Delta \tau$ correlation, b. $s-\Delta \tau_{D e t}^{1}$ correlation.

The contour lines represent the FHWM-levels. 
The observed arrival time distributions may be correlated with independent shower variables.

The correlation with muon multiplicity leads apparently to a considerable improvement of the separation of different primaries [19], but this feature is mainly an effect of the $N_{\mu} / N_{e}$ ratio alone, and with only little improvement by the additional information given by the arrival times.

More interesting appears to be the correlation with the shower ages (Fig. 11). The analysis reveals that in certain ranges of the shower age the arrival time distributions of the two different primaries are well separated, but nevertheless with strong overlap for the most frequent age values in a given $\mathrm{N}_{\mathrm{e}}$ range. As indicated the mass separation is improving with increasing size and energy of the air showers. This tendency gets more pronounced with larger distances $R_{\mu}$ from the shower center.

\section{Application of Bayes error estimation}

In order to explore the significance of differences observed for the muon arrival time distributions of EAS, induced by different primaries, we apply the recently introduced statistical techniques of estimation of the overlap of multivariate distributions and quantify the probability of a correct event classification or misclassification by the Bayes error. The mathematical basis and details of the method are described and illustrated in refs. 10 and 11. For sake of clarity we introduce the application with some general comments on the Bayesian inference technique.

\subsection{The Bayesian approach}

Analyses of experimentally observed phenomena of cosmic ray or particle physics are usually based on sufficiently accurate "model" calculations, predicting the values for a set of observables of a number of events of well specified physical origin and nature. Such calculations provide the so called "training samples" of labeled events, which implicitly reflect all correlations and variances of various event classes.

The models currently used in describing interaction and propagation processes in cosmic ray and particle physics, e.g., are very sophisticated and generate data by stochastic mechanisms. Such advanced models do no more parametrize the interesting quantities and their dependencies through explicitly 
given functional forms. Thus, the a-posteriori information and, in turn, the likelihood functions are provided in a nonparametric form.

Though Monte-Carlo simulations in cosmic ray physics are 'en vogue' and extensively used to predict the distributions of the observable quantities (and their fluctuations), originating from specified event classes, there are surprisingly few systematic studies to establish an efficient and statistically meaningful procedure of how to extract the optimum information from the actual data by comparing with the model prediction, given in a nonparametric form. For that a criterion of the closeness of multivariate distributions is needed. Since the extension of the standard one-dimensional methods meets serious difficulties, a straightforward procedure is a direct estimate of the overlap of the distributions in units of an error probability (with a value of 0.5 corresponding to full overlap of the distributions of different origin, and 0.0 to complete separation).

The strategy of statistical decisions of that kind is based on the Bayesian approach. It compares the a-posteriori probabilities (which associate an observed set $v$ to a definite event class, say for two different primaries - Fe and protons incident on the atmosphere), e.g. expressed by

$$
P(\text { proton } / v)>P(F e / v) \rightarrow d=\left\{\begin{array}{c}
F e \\
\text { proton }
\end{array}\right.
$$

The a-posteriori probabilities are estimated according to the Bayes theorem, e.g. written with the notation of our example of discriminating different primaries $\mathrm{Fe}$ and protons

$$
\begin{gathered}
P(F e / v)=\frac{P_{F e}}{P_{v}} P(v / F e) \\
P(\text { proton } / v)=\frac{P_{\text {prot }}}{P_{v}} P(v / \text { proton }) .
\end{gathered}
$$

Here $\mathrm{P}(u / \mathrm{Fe})$ are the conditional probability densities (likelihood functions) inferred from the correspondent training samples, $P_{F e}$ and $P_{\text {prot }}$ are the a-priori probabilities, i.e. the initial assumption about the probabilities to "detect" specific types of events among all events $\left(\mathrm{P}_{v}\right)$. The consistency of such initial assumptions is just the matter to be improved by iterative application of Bayes theorem.

Several procedures are available to prepare the likelihood functions $(\mathrm{P}(\mathrm{v} / \mathrm{Fe})$ e.g.) from the results of Monte-Carlo simulations, including neural network techniques. We use here the most popular Parzen window [21] procedure which 
substitutes each element of the training sample by bell-shaped functions and constructs the probability density by a superposition of many "kernels" in the feature space, centralized around each point in the training sample (for details see refs. 21 and 22).

The chance to misclassify a particular observed set $v_{a}$ equals to

$$
r\left(v_{a}\right)=\operatorname{Min}\left\{P\left(\text { proton } / v_{a}\right), P\left(F e / v_{a}\right)\right\} .
$$

The mathematical expectation of this probability taken over the measurement space $V$ is the Bayes error

$$
R=\int_{V} r(v) P(v) d v .
$$

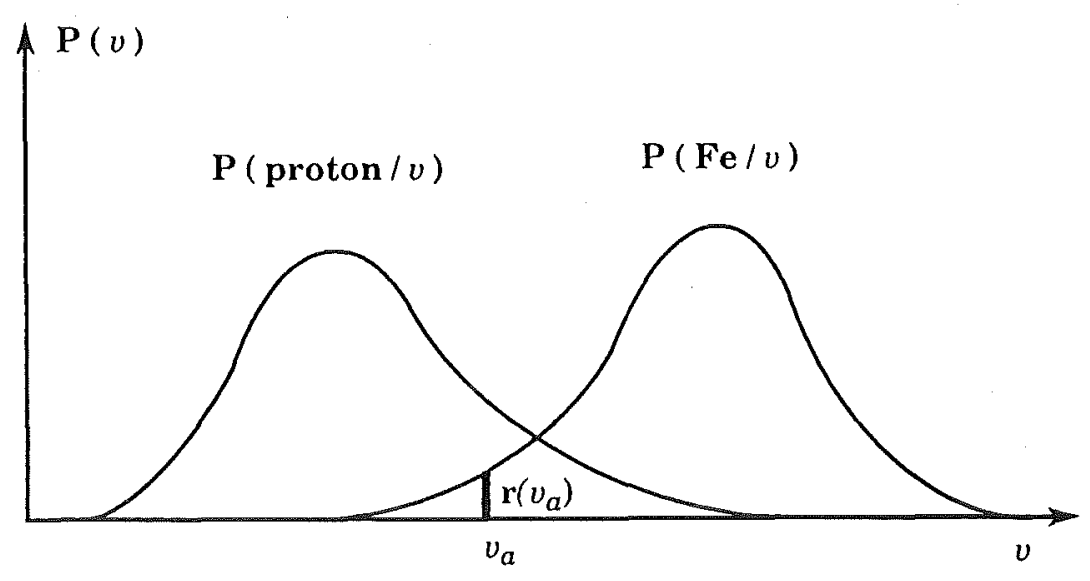

Fig. 12: A-posteriori probabilities and Bayes error definition.

There are various different methods for estimating the Bayes error [23]. We apply here the rather efficient "one-leave-out-for-a-time" test which removes one element from the sample and trains the sample without it. The element is subsequently classified and returned to the sample. The procedure is then repeated till all elements are classified [24]. An error estimate $R^{e}$ may be performed by introducing a random variable.

$$
\varepsilon(v)=\left\{\begin{array}{l}
0, \text { if classified correctly } \\
1, \text { otherwise }
\end{array}\right.
$$

and counting the number of errors during the test 


$$
R^{e}=\frac{1}{M} \sum_{i=1}^{M} \varepsilon\left(v_{i}\right)
$$

Another kind of estimate, the "average conditional error $R^{p}$ " is directly related to the definition of Bayes error $R$

$$
R^{p}=\frac{1}{M} \sum_{i=1}^{M} \operatorname{Min}\{P(\text { proton } / v), P(F e / v)\}
$$

Both error estimates can be shown to have slightly different variances [25].

We note that the quantities introduced for conclusive comparisons are not directly related to quantities (like rms error etc.) of significance in standard statistical interpretations.

The application of the full approach is a two-step procedure, first the selection of presumably relevant experimental features (viewed as an optimization problem) and then a classification with Bayes decision rule. In this paper we do not discuss the methods of optimal subset selection, we just consider the muon arrival time distributions (provided by Monte-Carlo simulations) and their correlations with other EAS observables, and compare the results in terms of the values of the resulting Bayes errors.

\subsection{Results of the analysis}

Figs. 12a,b,c,d display the results of the "One-leave-out-for-a-time" test: the classification probabilities and the Bayes errors for the cases (corresponding to the distributions shown in Figs. 2, 9, 10, 11) of the "theoretical" distributions, of the mean muon delay time $(\Delta \tau)$ distributions, of the $\Delta \tau^{1}{ }_{\text {Det }}$ distributions and of the $\Delta \mathrm{r}_{\text {Det }}^{1}$ - shower age correlation for the $\mathrm{R}_{\mu}$-bin $100-150 \mathrm{~m}$ from the shower axis. The tendency of improving the seperation with increasing $\mathrm{N}_{\mathrm{e}}$ is quantified. We note the slight improvement by the correlation with the shower age. 

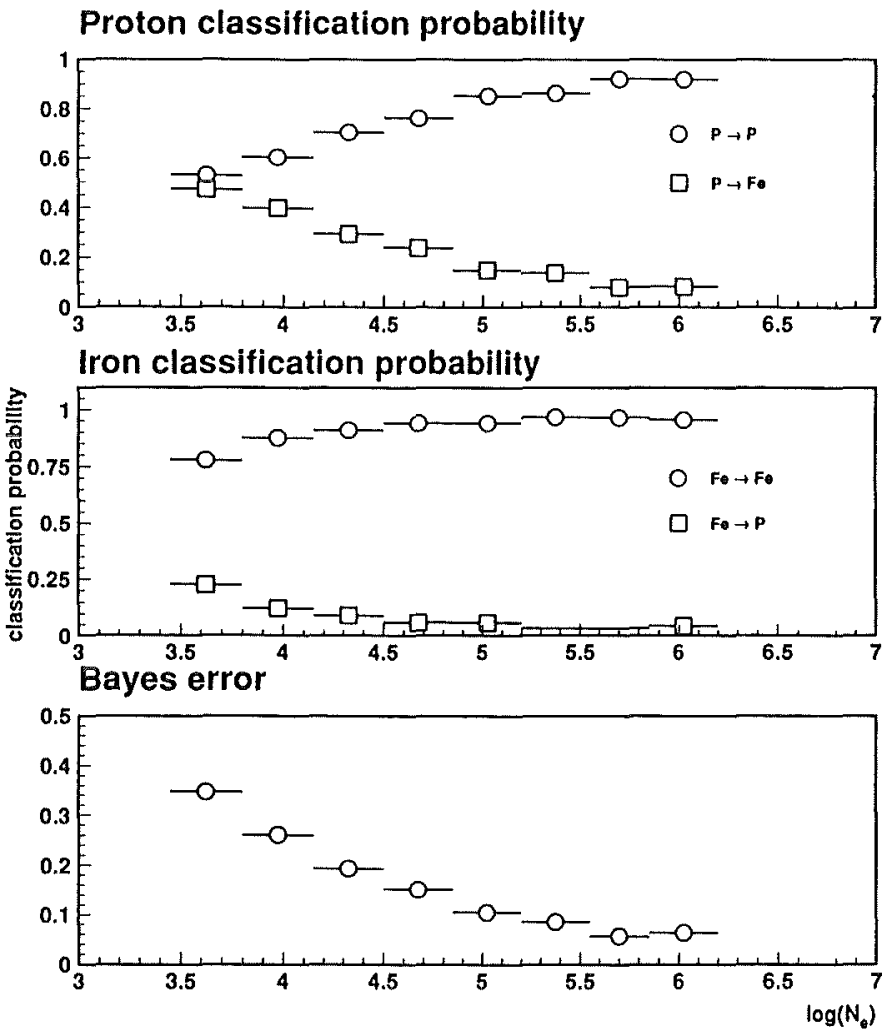

Fig. 13 a:

Classification

probabilities for

the mean arrival

times: "theo-

retical" distri-

butions.

Fig. 13 b:

Proton classification probability

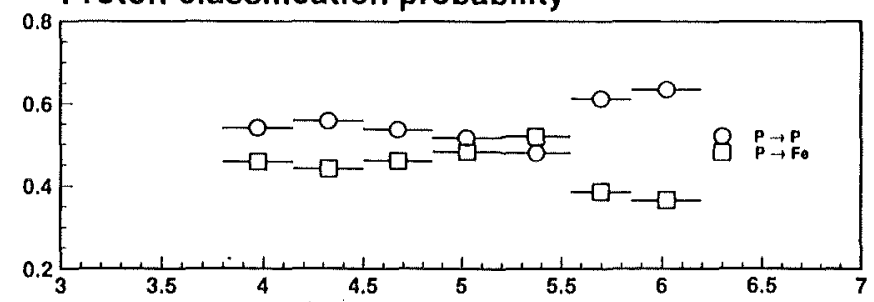

Iron classification probability

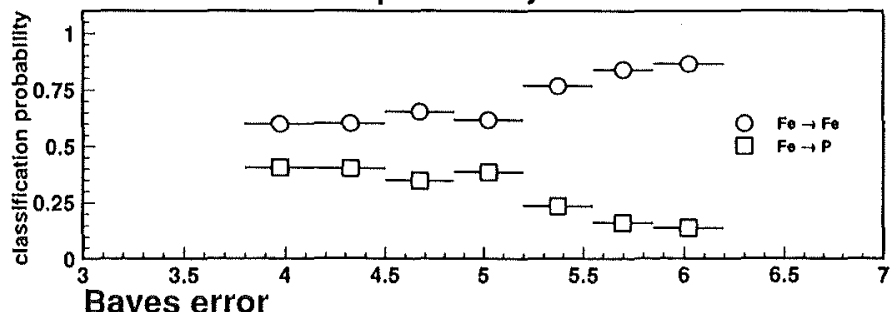

\section{Classification}

probabilities for

the mean arrival

times: mean muon delay time distributions

$(\Delta \tau)$.

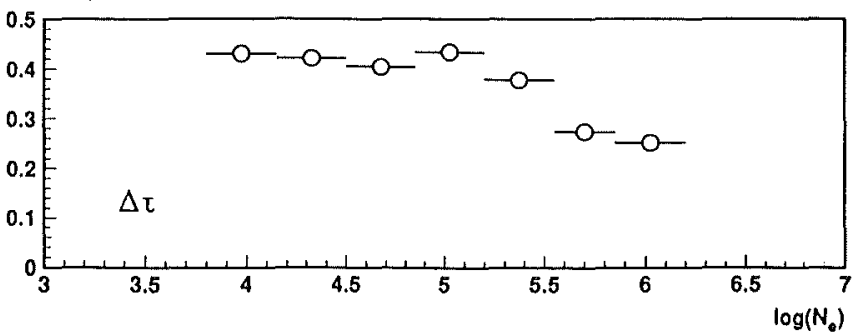



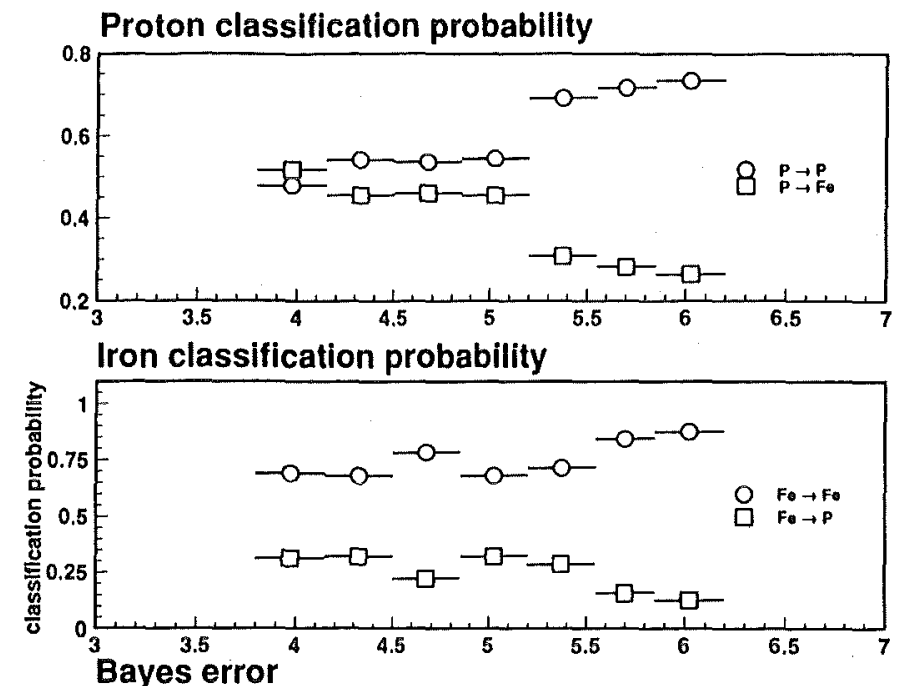

Fig. 13 c:

\section{Classification}

probabilities for

the mean arrival

times: arrival

time distribu-

tions of the

foremost muon

$\left(\Delta \mathrm{\tau}^{1}{ }_{D e t}\right)$.

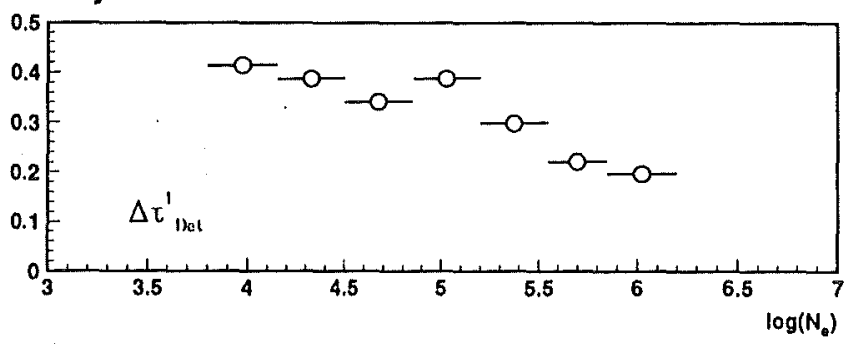

Fig. 13 d:

Classification

probabilities for

the $\Delta \tau^{1}{ }_{D e t}-$

shower age

correlation.

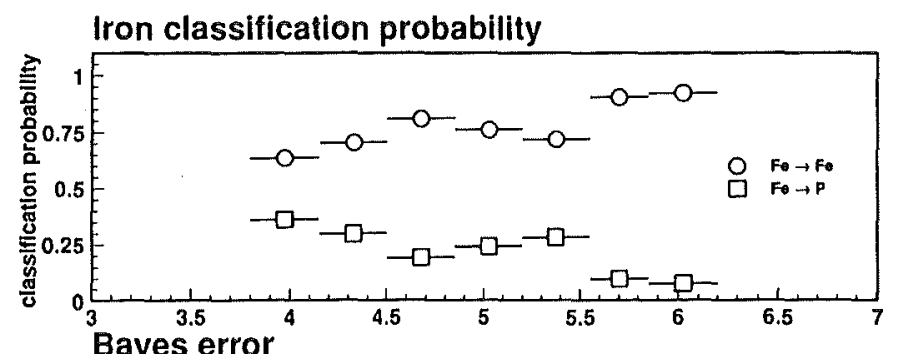

Bayes error

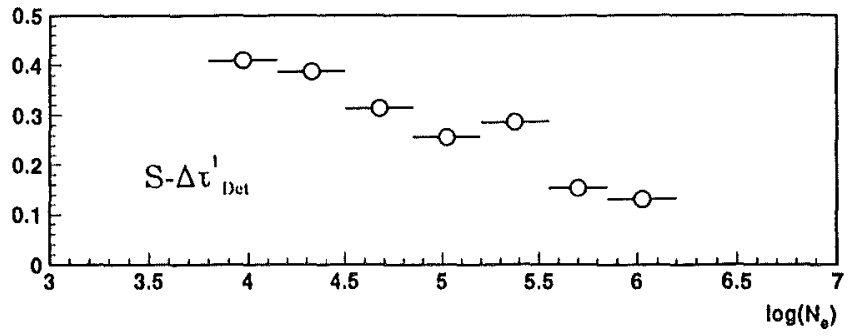




\section{Concluding Remarks}

The present analysis tried to explore the information of arrival time distributions of EAS muons, which can be measured with the timing facility of the KASCADE central detector. Provided that the measurements with the KASCADE array will establish the basic EAS information about sizes $N_{e}$, core location, direction of incidence and arrival time of the shower front with sufficient accuracy, the arrival time distributions of the muons, registered in an adequate distance of the shower axis, exhibit promising signatures of the mass composition, increasingly for larger shower sizes, i.e. higher energies, around $1016 \mathrm{eV}$.

As compared to "theoretical" distributions (based on a compete collection of the total number of muons in a particular $R_{\mu}$-bin) the deterioration of the signal by the finite size of the detector and the low muon density is clearly significant.

It should be noted that the present analysis does not invoke additional information available from a correlation with muon multiplicity $N_{\mu}$. Just for larger incident energies with larger extent of the muon lateral distributions, $N_{\mu}$ might be poorly determined (at least if the analysis is not biased by a prechosen analytical form). Using the correlation with shower age leads to a slight improvement of the mass discrimination, especially for larger shower sizes.

The most significant signal is provided by the relative arrival time of the foremost muon $\left(\Delta \tau^{1} \mu\right.$ and $\Delta \tau^{1}{ }_{\text {Det }}$, respectively) which obviously preserves the information of the first interactions better than the mean value $\Delta \tau$. Since these time signals are both referred to the arrival time of the shower core, they reflect also differences in the shape of the lateral distribution $\rho_{\mu}\left(R_{\mu}\right)$ for different primaries at the same energy, thus being sampled at a particular distance $R_{\mu}$, and with a prechosen muon energy detection threshold.

The authors acknowledge valuable suggestions and contributions of $V$. Corcalciuc, A.D. Erlykin, T. Thouw and K.W. Zimmer. We are also grateful to D. Heck and J. Knapp for the help in using the CORSIKA simulation code. 


\section{References:}

[1] J. Linsley and L. Scarsi, Phys. Rev. 128 (1962), 2384

[2] P.K.F. Grieder, Proc. 17th ICRC, Paris 6 (1981), 288

[3] M.L. Armitage, P.R. Blake and W.F. Nash, Journ. Phys. A: Math. Gen. 8 (1975), 1005

P.R. Blake, M. Luksys, W.F. Nash and A.J. Sephton, Proc. 19th Int. Conf. on Cosmic Rays, La Jolla 7 (1985), 123 and 127

[4] P.R. Blake, V.S. Collis, M. Luksys, W.F. Nash and A.J. Sephton, Journ. Phys. G: Nucl. Part. Phys. 16 (1990), 755

[5] F. Kakimoto, T. Tamura, Y. Matsumoto, T. Enoki, I. Tsuchimoto, K. Nishi and K. Suga, Journ. Phys. G: Nucl. Phys. 12 (1986), 151

[6] E.J. Villiers, D.J. van der Walt, P.K.F. Grieder and G. van Urk, Journ. Phys. G: Nucl. Phys. 12 (1986), 547

[7] G.B. Kristiansen et al, Proc. 21st Int. Conf. on Cosmic Rays, Adelaide (Australia) 9 (1990), 150

[8] P. Doll et al., KfK-Report 4886 (1990), Kernforschungszentrum Karlsruhe - Nucl. Phys. B (Proc. Suppl.) 14A (1990), 336

[9] H. Rebel and the KASCADE Collaboration, Proc. VII, Intern. Symposium on Very High Energy Cosmic-Ray Interactions, Ann Arbor (Michigan), June 21-27, 1992 - AIP Conference Proceedings 276, ed. L. Jones

[10] A. A. Chilingarian, Computer Physics Comm. 54 (1989), 381

[11] A. A. Chilingarian and G. Z. Zazian, Nuovo Cim. 14 (1991), 555

[12] J.N. Capdevielle et al., KfK-Report 4998 (1992), Kernforschungszentrum Karlsruhe

[13] J.N. Capdevielle, Journ. Phys. G: Nucl. Part. Phys. 15 (1989), 909

[14] A. Capella and J. Tran Thank Van, Phys. Lett. B93 (1980), 146

[15] W.R. Nelson, SLAC Report 265 (1985)

[16] H. Fesefeldt, Report PITHA 85/02, Physikalisches Institut der TH Aachen, Germany, 1985 
[17] D. Heck and G. Schatz, in KfK-Report 5027 (1992), Kernforschungszentrum Karlsruhe, p. 35 - eds. J. Knapp and H.Rebel

[18] T.V. Danilova, D. Dumora, A.D. Erlykin and J. Procureur, Journ. Phys. G: Nucl. Part. Phys. 20 (1994), 961

[19] M. Brendle, A. Hanser, G. Völker and H. Rebel, KfK-Report 5027 (1992), p. 29, eds. J. Knapp and H. Rebel, Kernforschungszentrum Karlsruhe

[20] H. Rebel, in "Topics in Atomic and Nuclear Collisions", NATO ASI Series B321, eds. B. Remaud, A. Calboreanu and V. Zoran, Plenum Press 1994, p. 397; - Proc. Intern. Conf. Astrophysics and Cosmology: Birth Centenary Celebration of M.N. Saha, Dec. 20-23, 1993, Saha Institute of Nucl. Physics, Calcutta, India, eds. R.K. Moitra and B.K. Sinha

[21] E. Parzen, Ann. Math. Stat. 33 (1962), 1065

[22] P.C. Maholonobis, Proc. Nat. Inst. of India 2 (1936), 49

[23] G.T. Toussaint, IEEE Trans. on Information IT-20 (1974), 472

[24] S.M. Snappin and J.D. Knoke, Technometrics 26 (1984), 371

[25] K. Fukunaga and D. Himmels, IEEE Trans. on Pattern Analysis and Machine Intelligence, PAMI 9 (1987), 634 


\section{Appendix: Display of various features of the arrival time distributions}

The following figures extend the display of various features of the time arrival distributions discussed in the preceding chapters.

Fig. A1: Mean muon arrival time distributions for various ranges of the shower size and different radial distances from the shower axis of proton and iron induced air showers (observed at sea level).

(Time zero: Time of the first interaction $\mathrm{T}^{1}{ }_{\text {int }}$ )

Fig. A2: Mean muon delay time distributions for various ranges of the shower size and different radial distances from the shower axis of proton and iron induced air showers (observed at sea level).

(Time zero: Arrival of the shower core $\tau_{c}$ )

Fig. A3: Generation origin of the arriving muons:

Mean arrival time distributions decomposed by the origin of the foremost muon.

Fig. A4: Generation origin of the arriving muons:

Mean arrival time distributions decomposed by the median generation of the events.

Fig. A5: Local mean arrival time distributions including detector geometry, response and time-resolution for an event multiplicity $\geqq 5$.

Fig. A6: Mean muon delay time distributions including detector geometry, response and time-resolution for the event multiplicity $\geqq 5$.

Fig. A7: Delay time distributions of the foremost muon including detector geometry, response and time-resolution for the event multiplicity $\geqq 5$.

Fig. A8: Muon lateral distributions for $\mathrm{Fe}$ and $\mathrm{p}$-induced extensive air showers at $\mathrm{E}_{0}=1016 \mathrm{eV}$. 


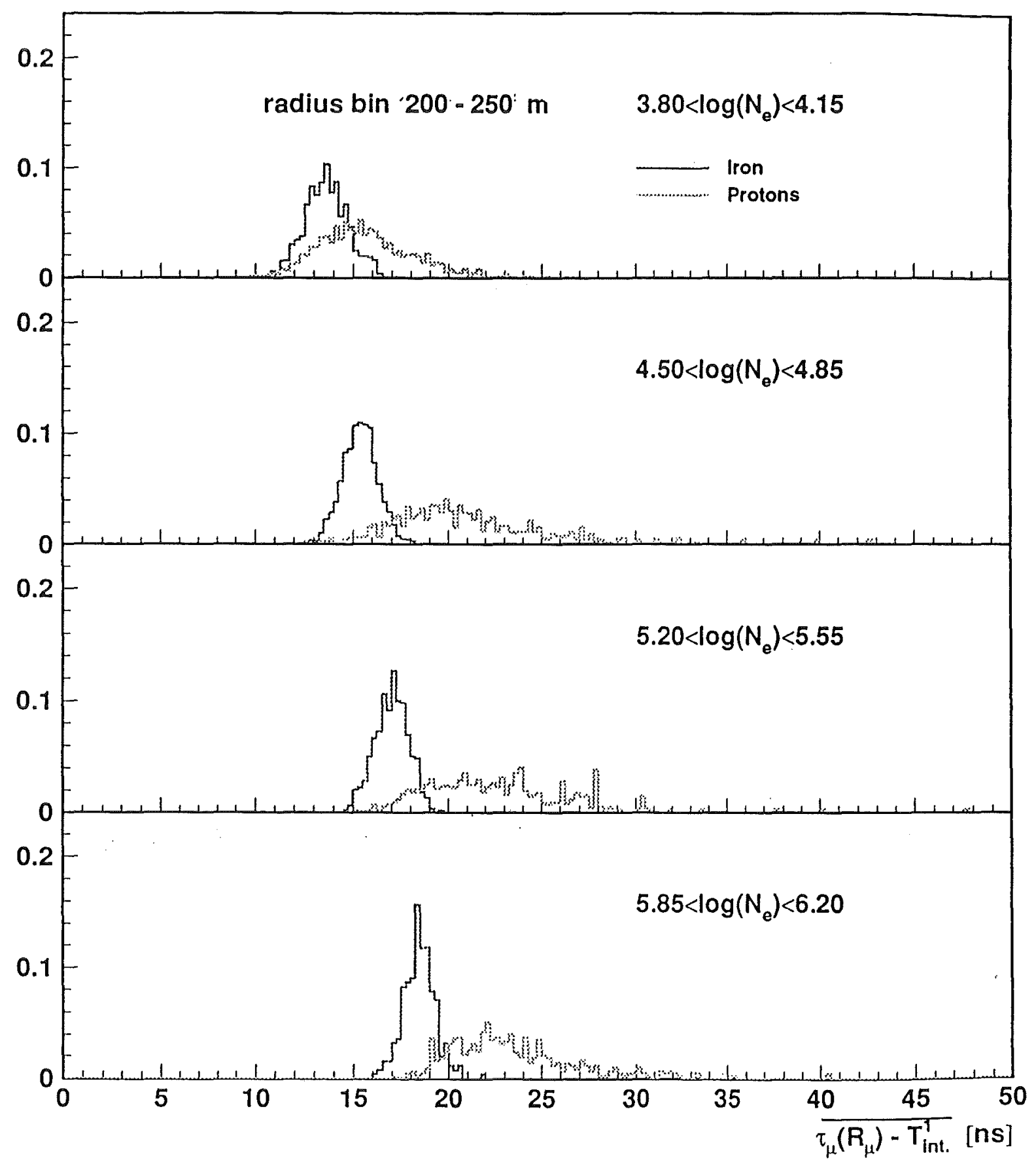

Fig. A1 


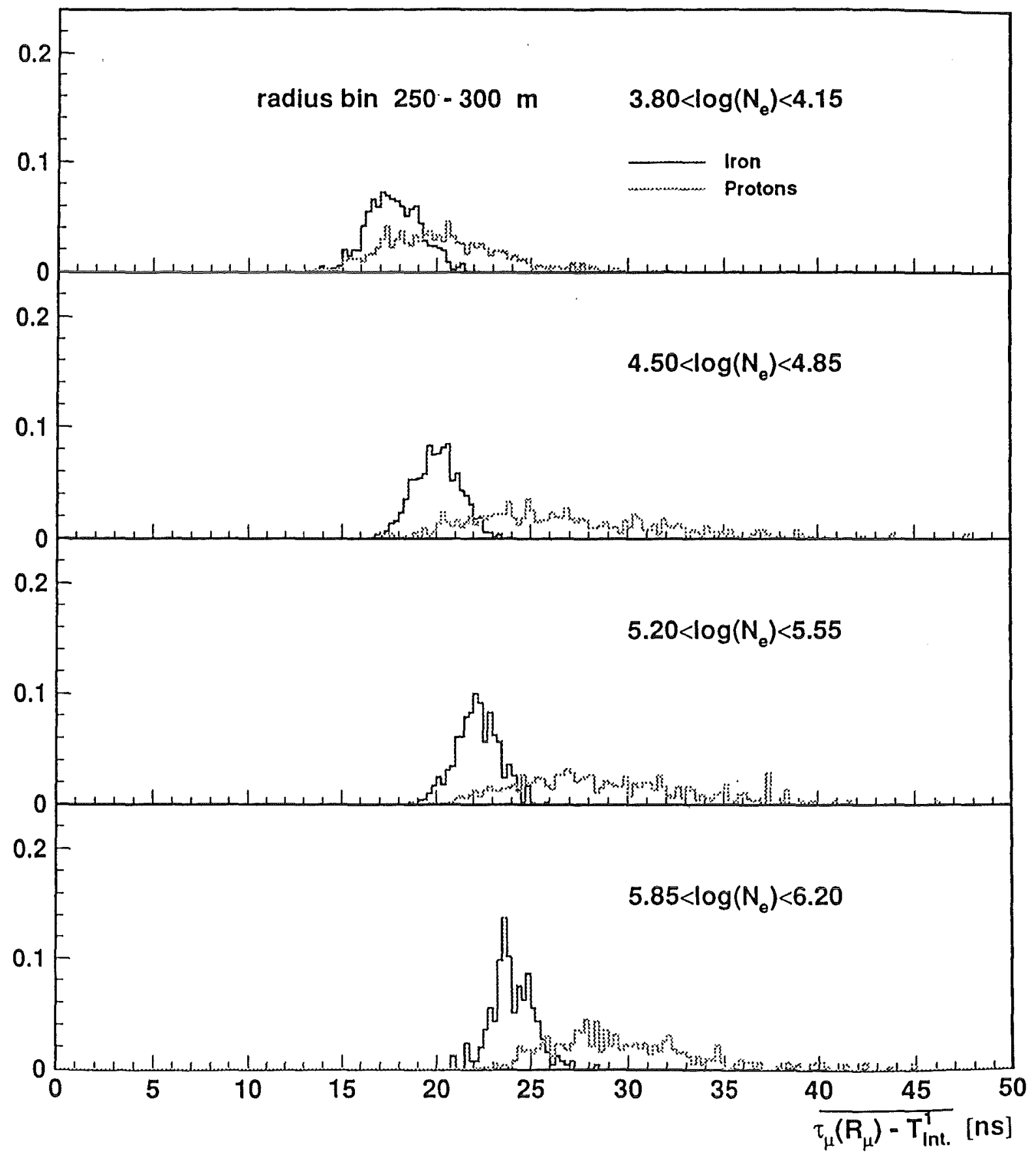

Fig. A1 


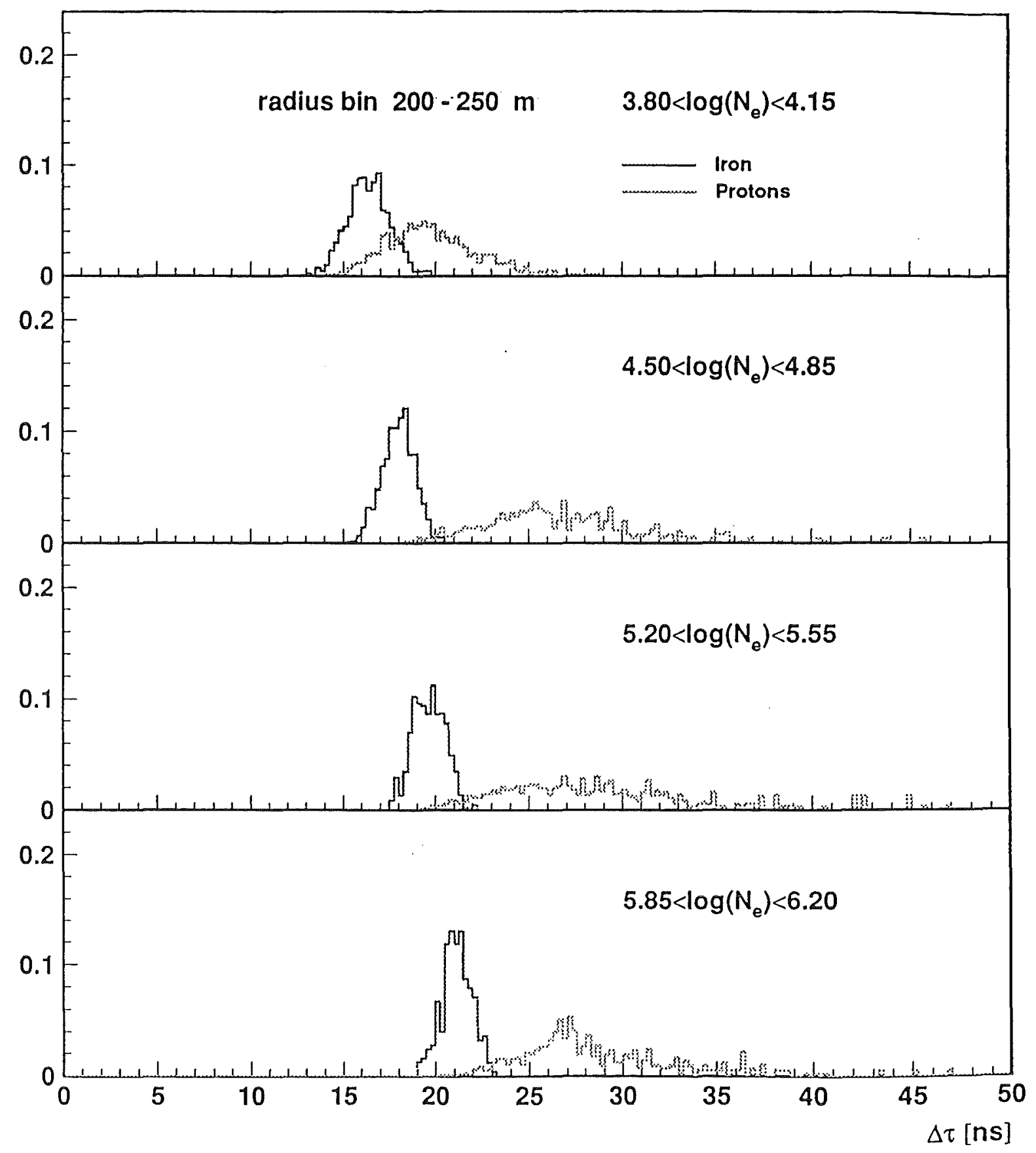

Fig. A2 


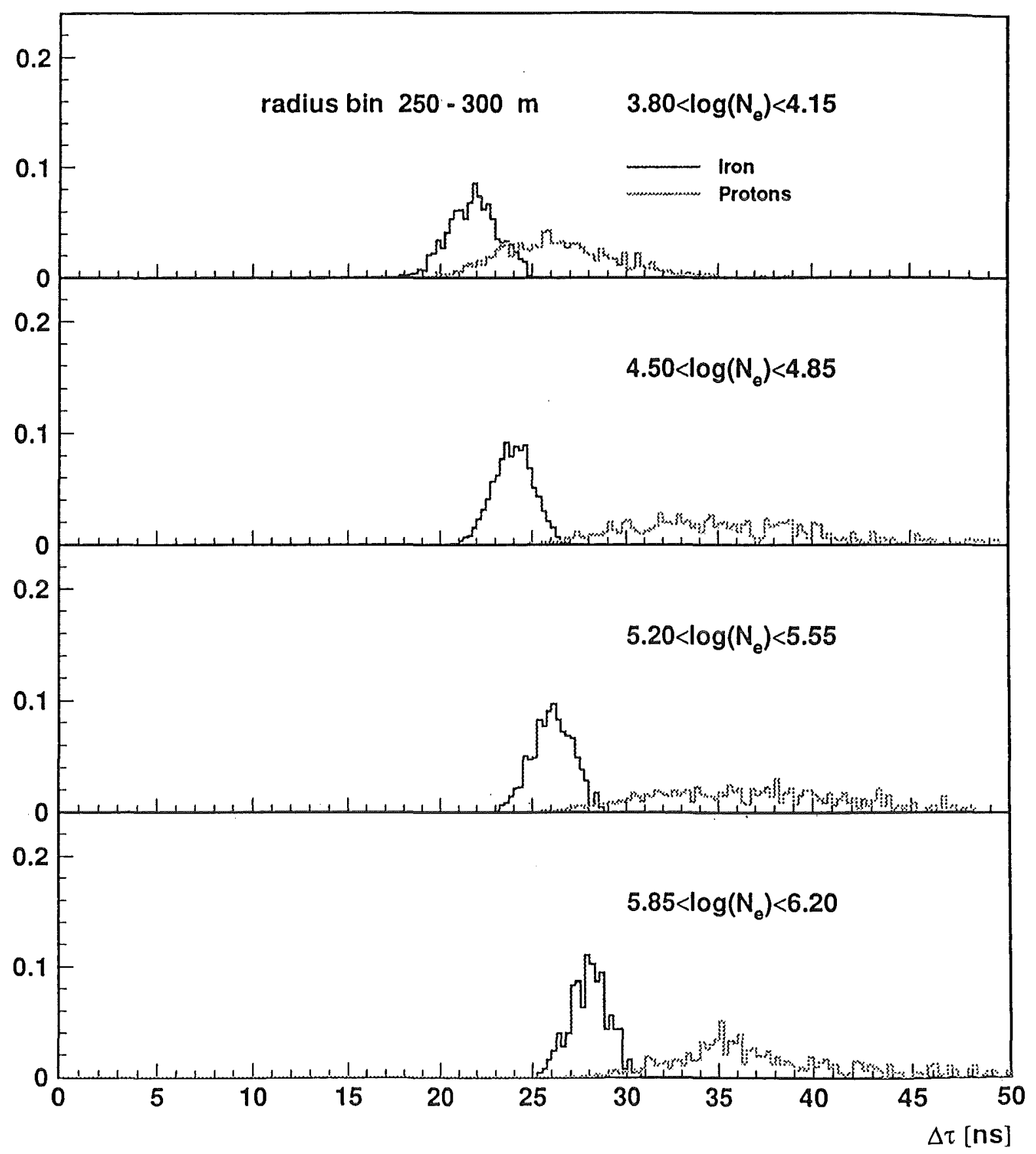

Fig. A2 

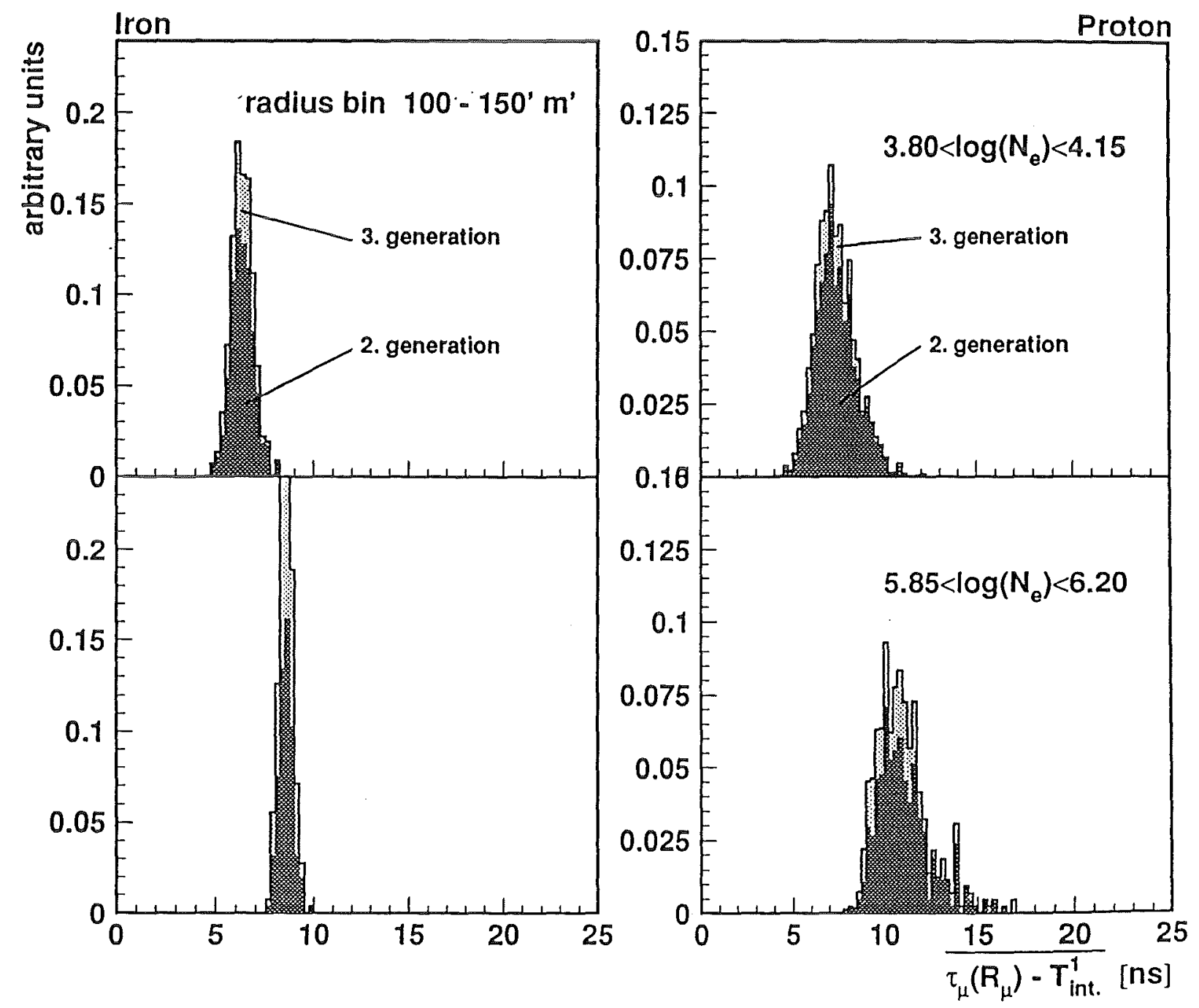

Fig. A3 


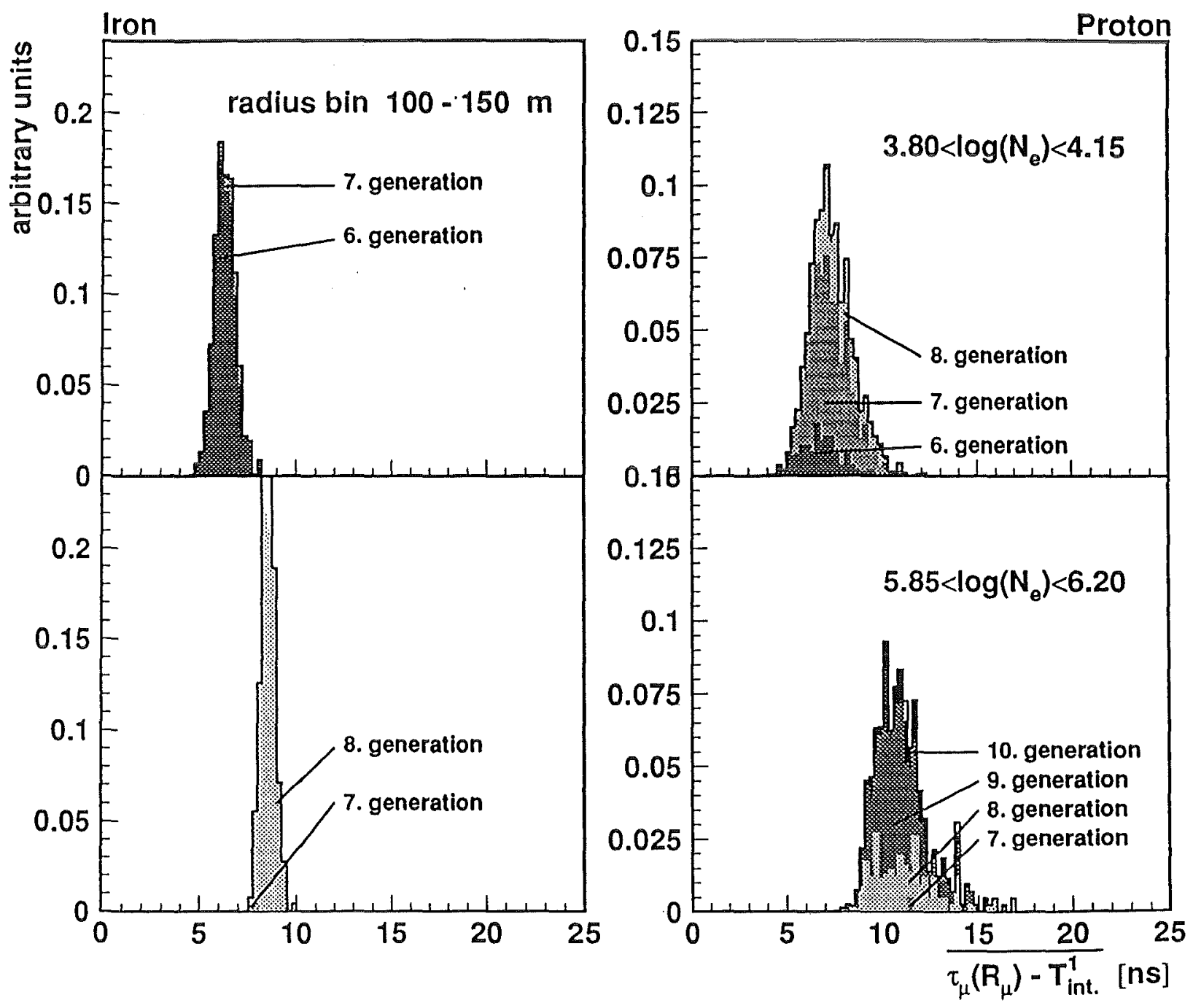

Fig. A4 
Mean arrival time including detector geometry

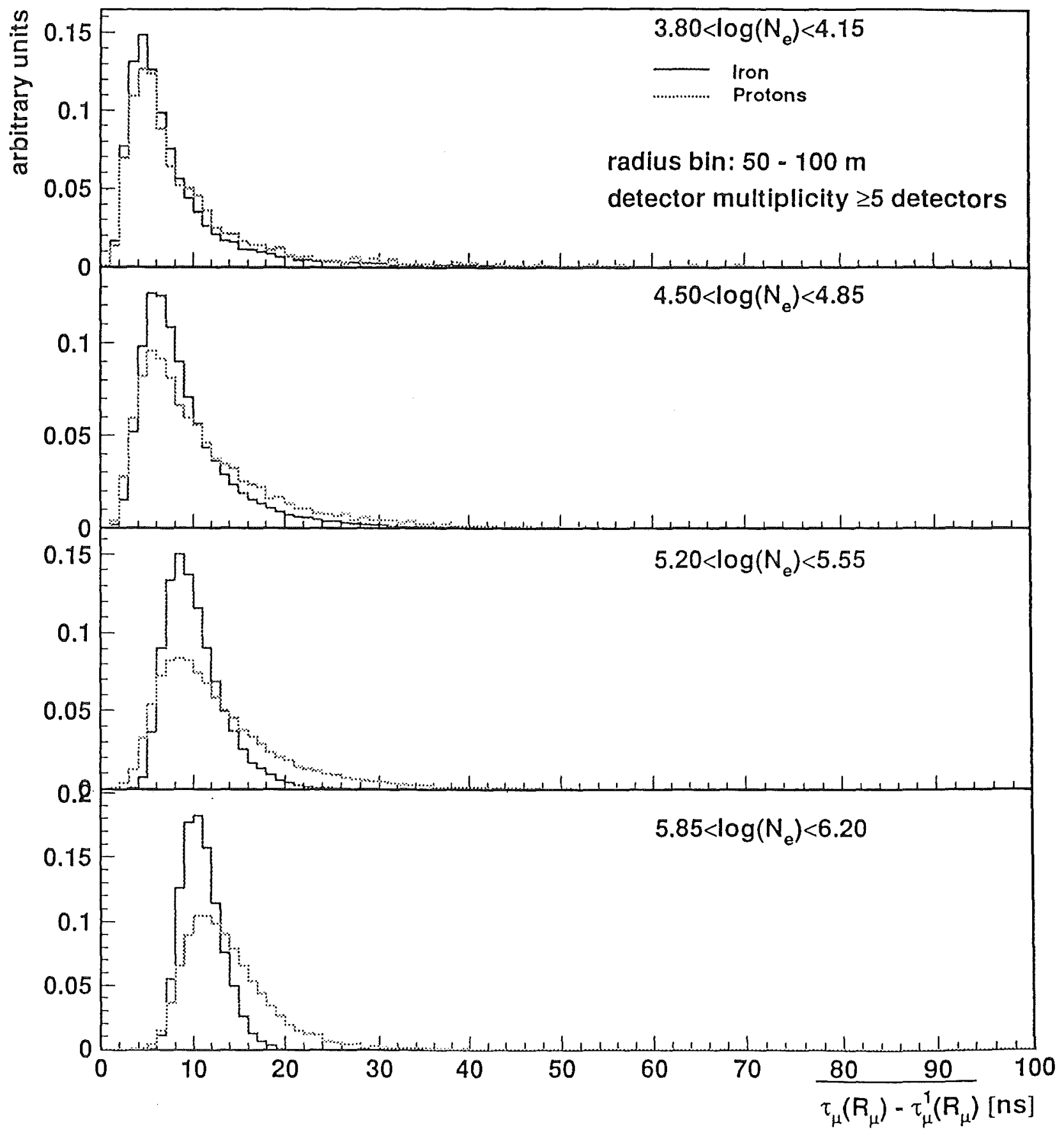

Fig. A5 
Mean arrival time including detector geometry

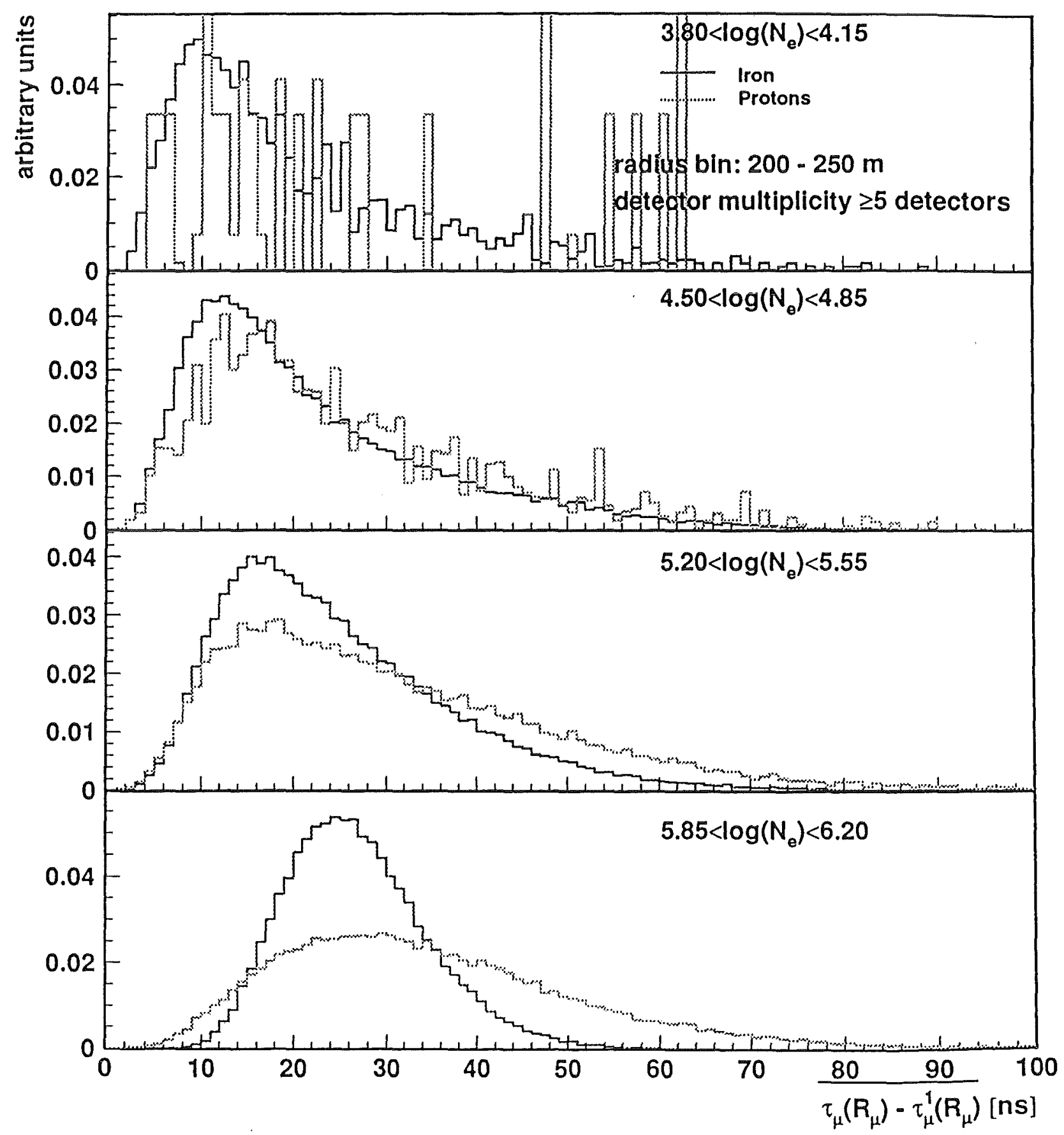

Fig. A5 
Mean arrival time including detector geometry

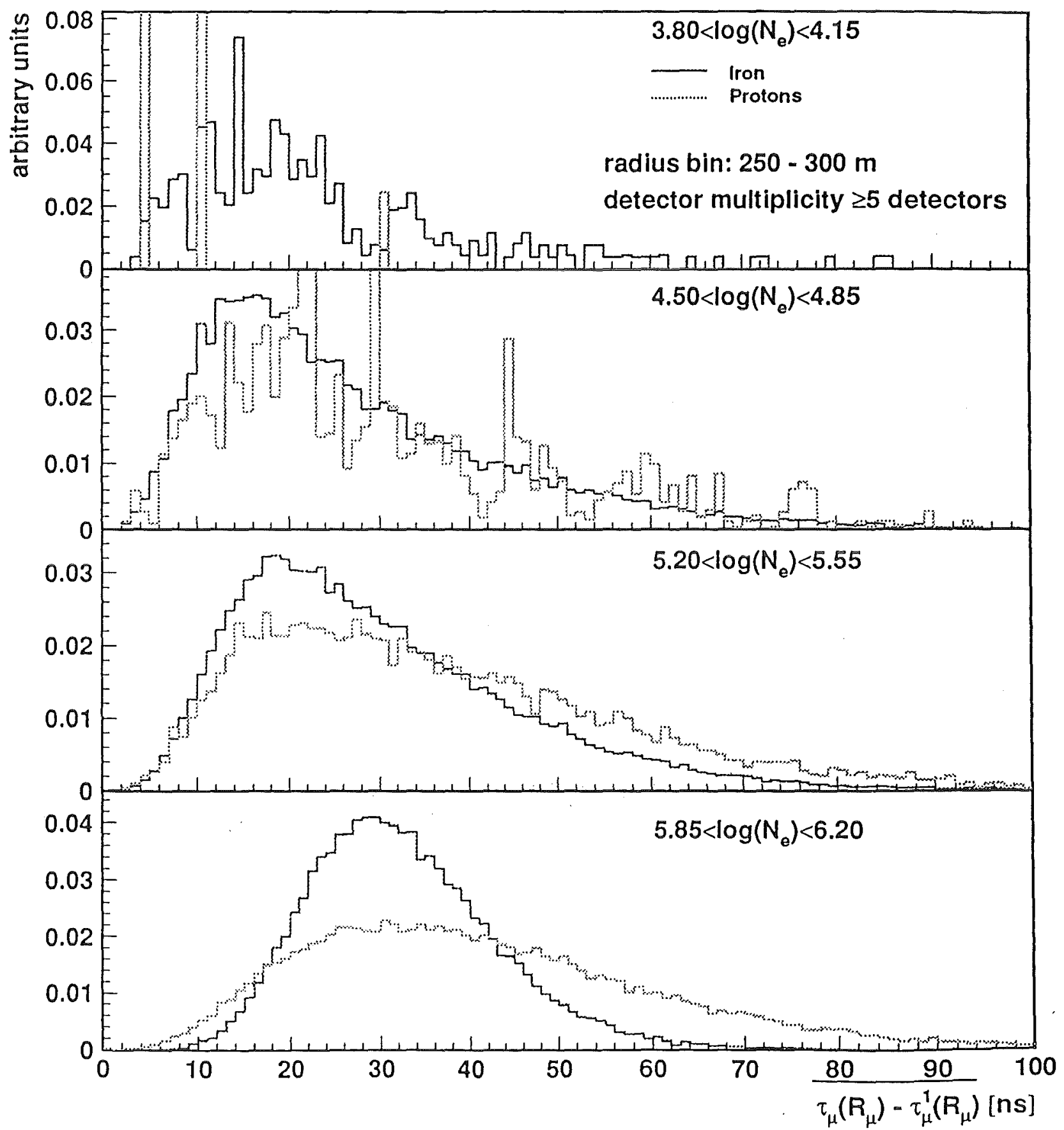

Fig. A5 
Mean arrival time including detector geometry

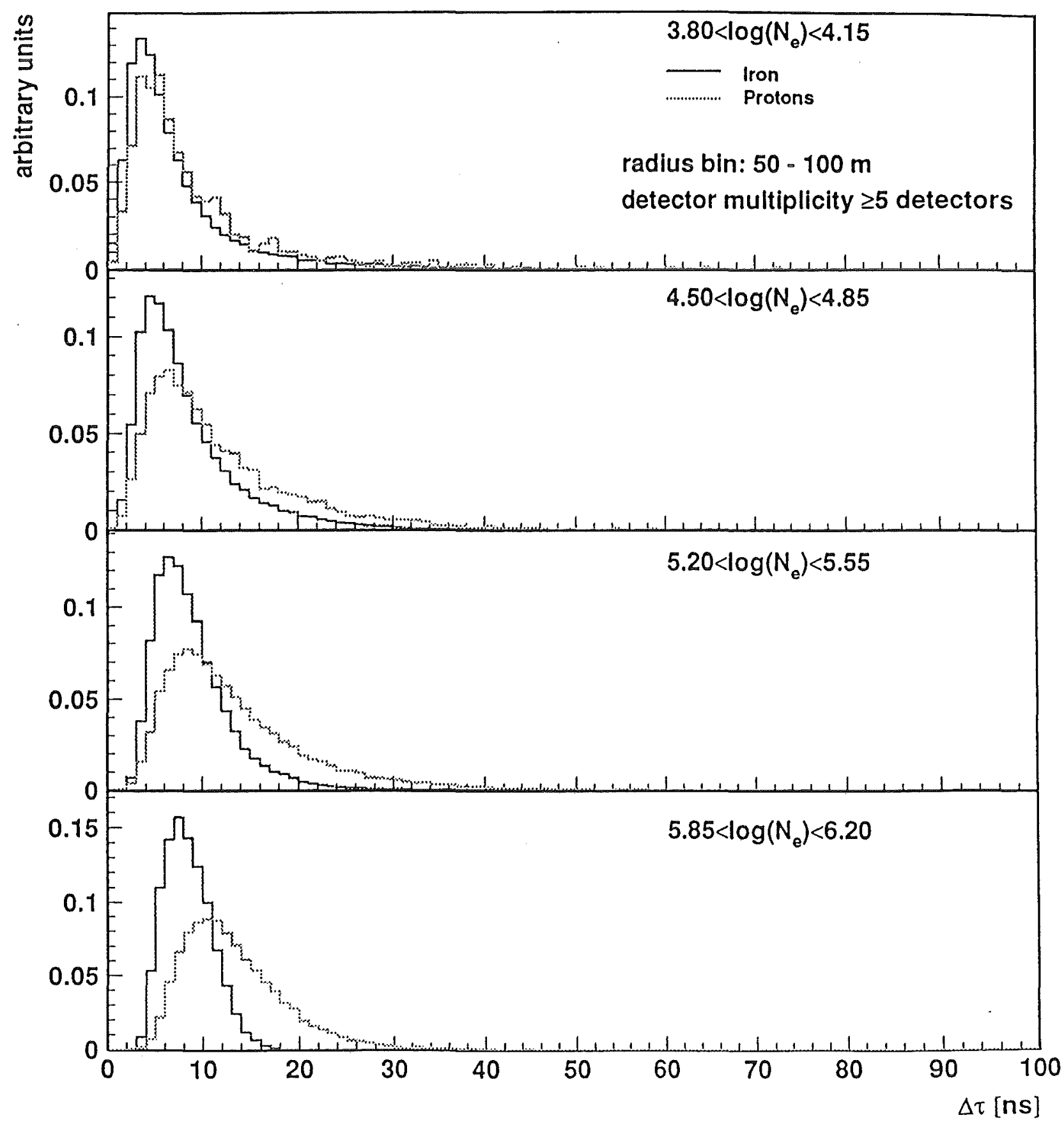

Fig. A6 
Mean arrival time including detector geometry

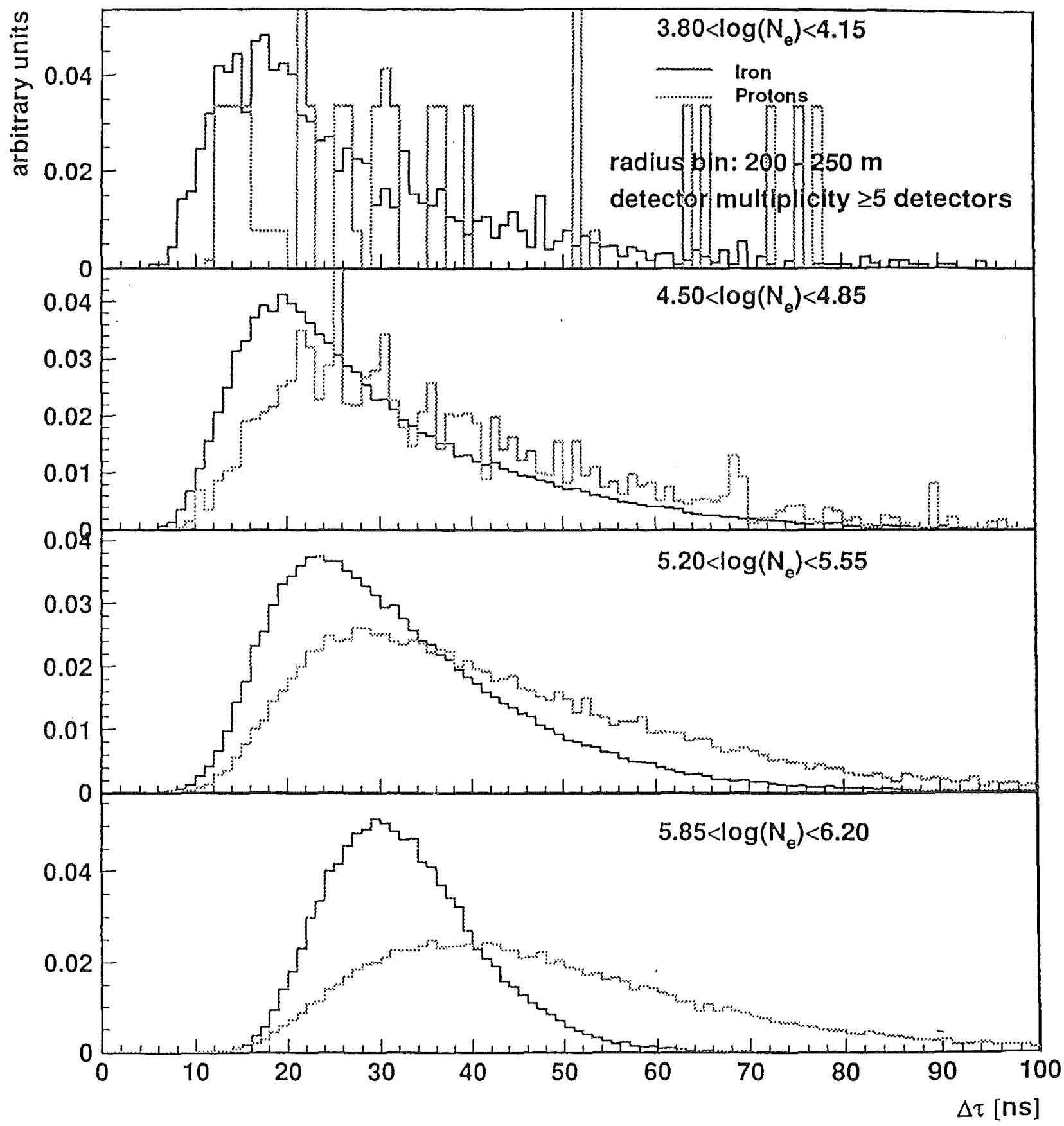

Fig. A6 


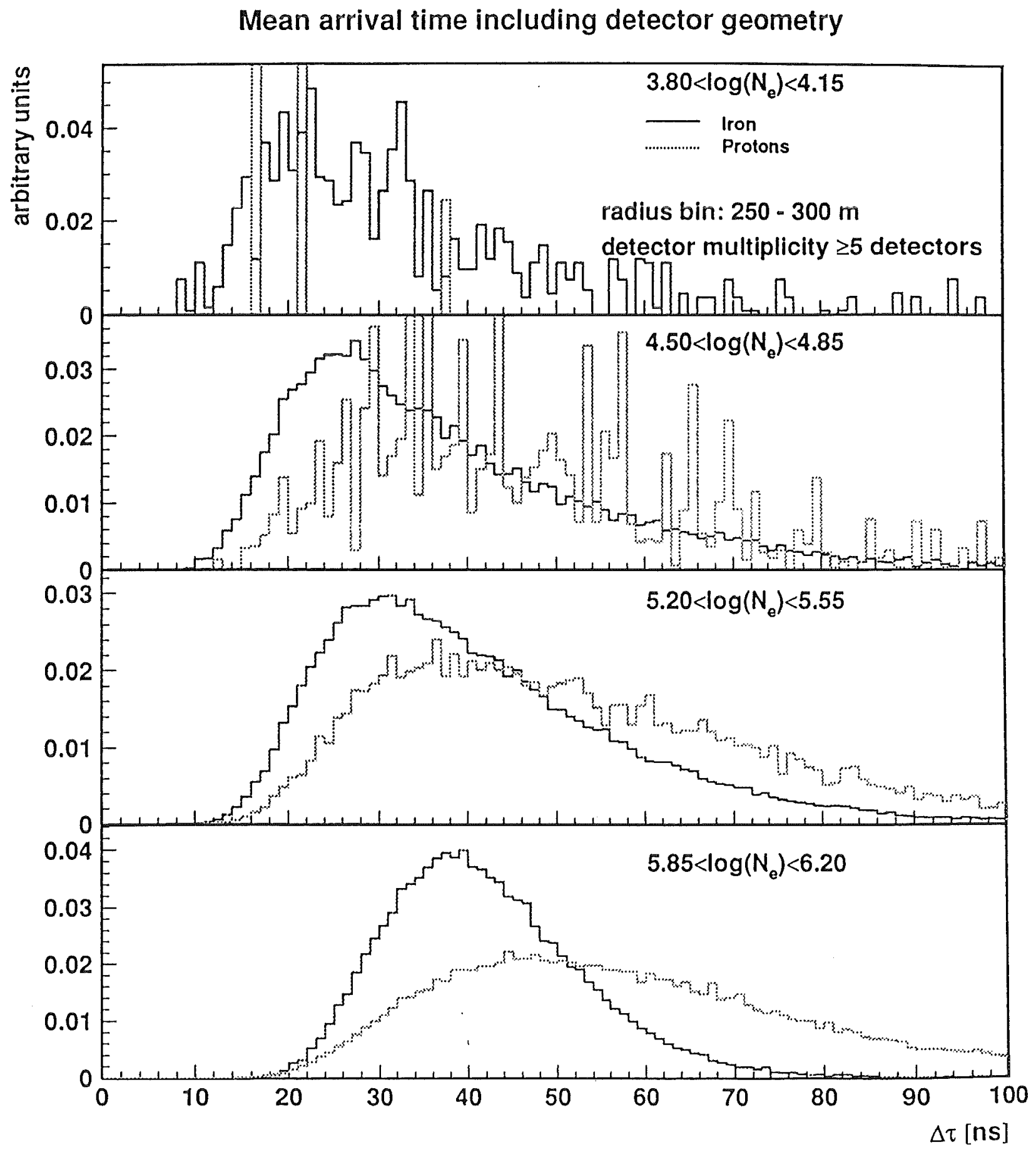

Fig. A6 
arrival time of first detector including detector geometry

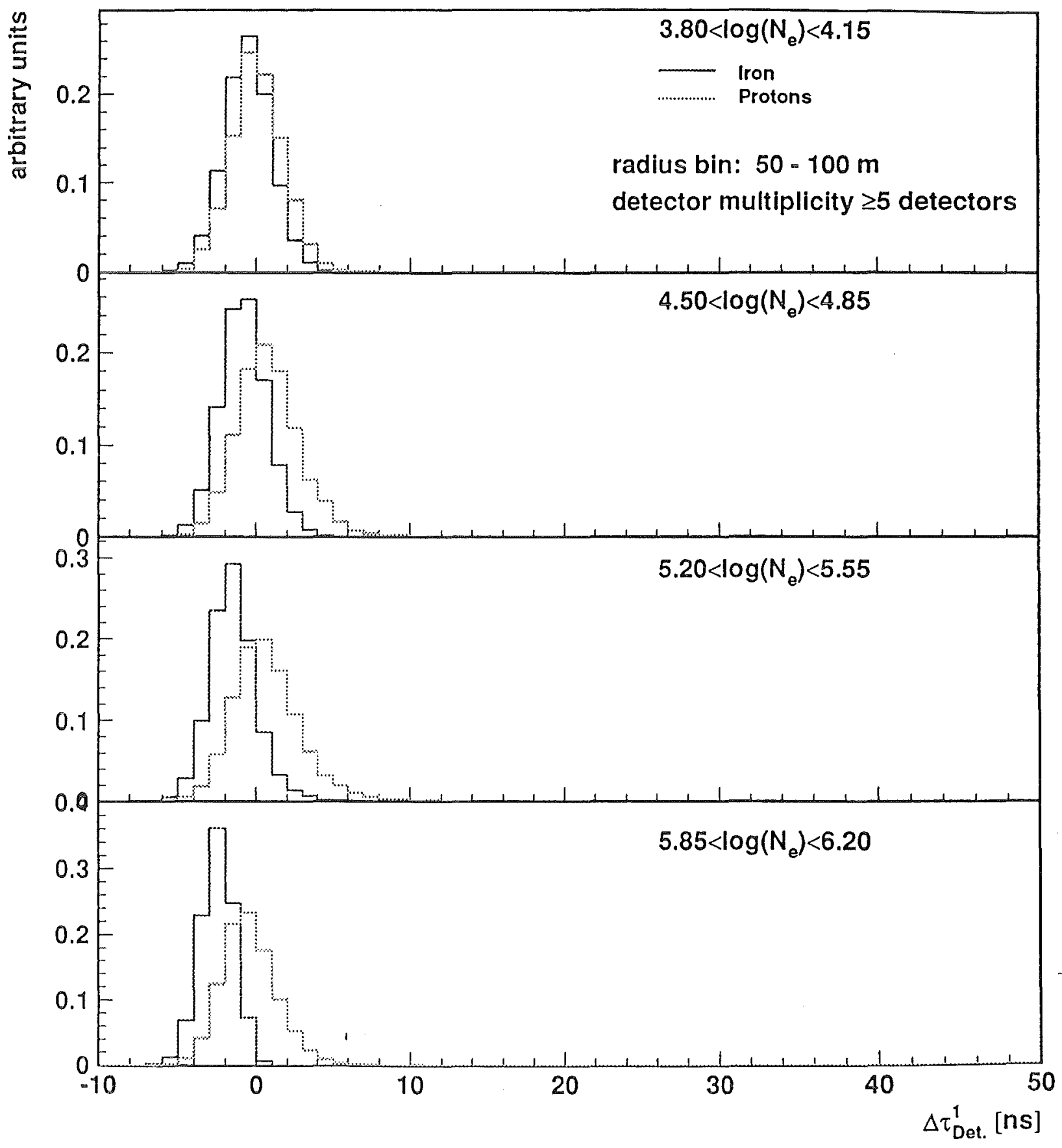

Fig. A7 
arrival time of first detector including detector geometry

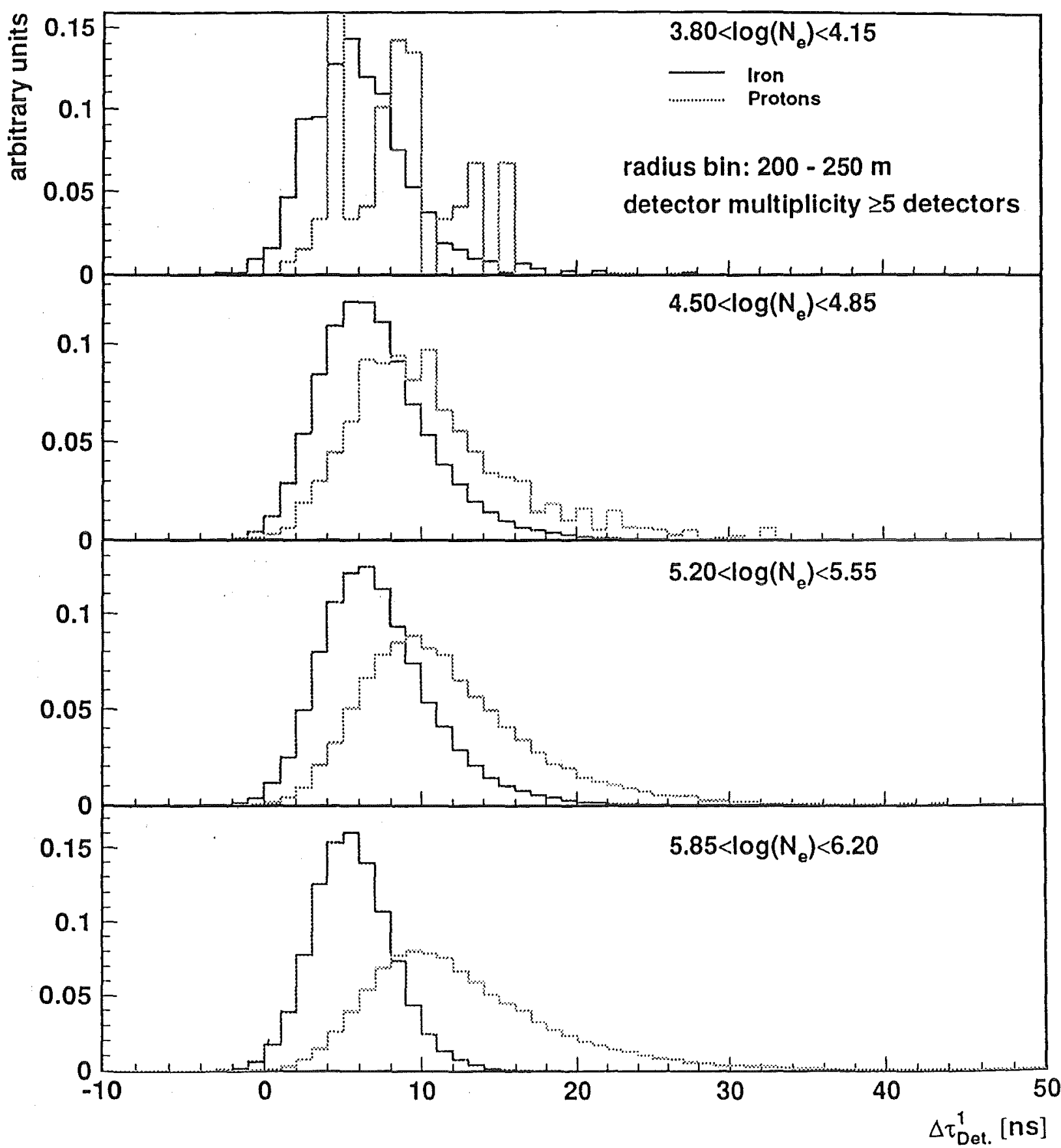

Fig. A7 
arrival time of first detector including detector geometry

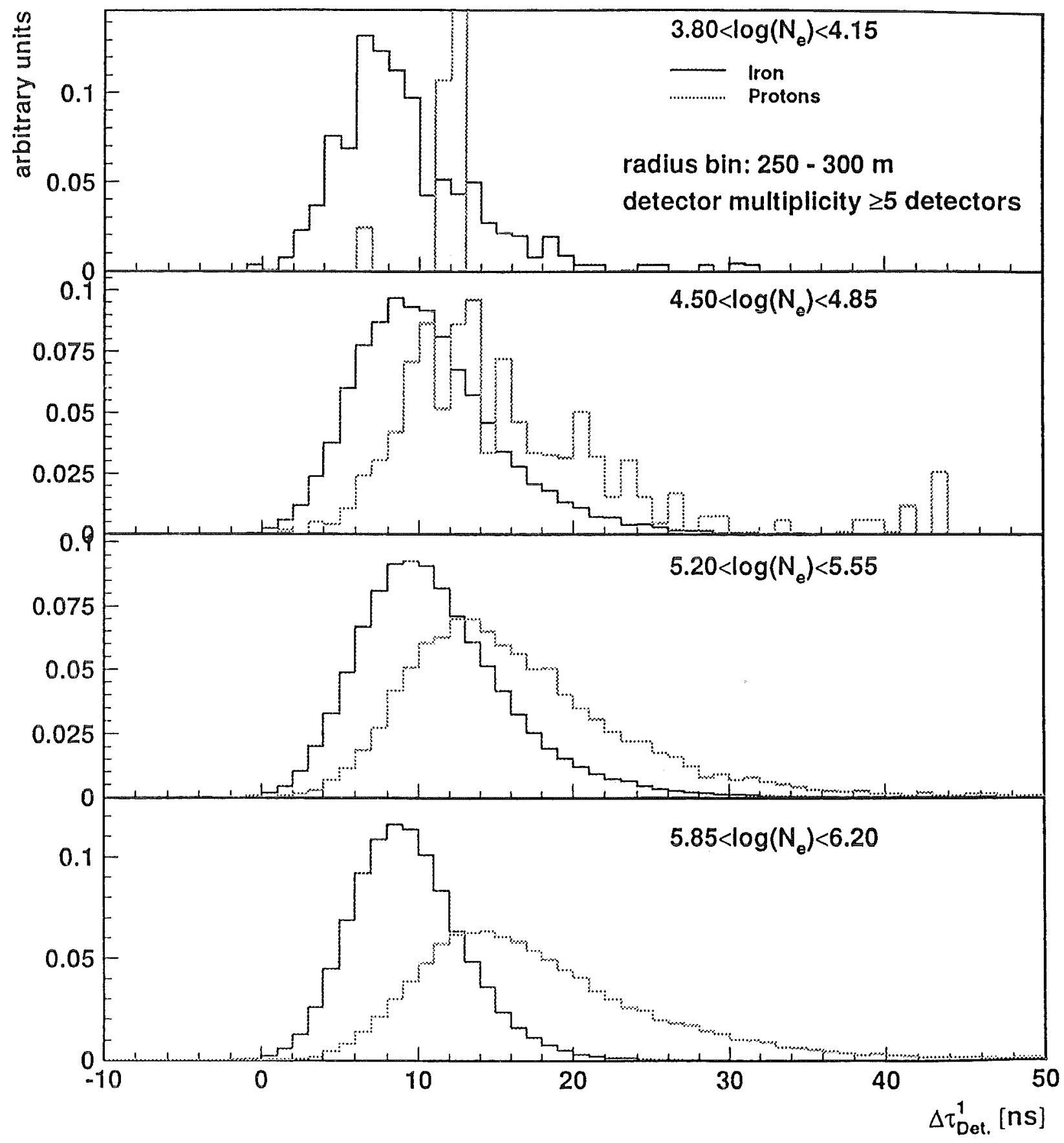

Fig. A7 


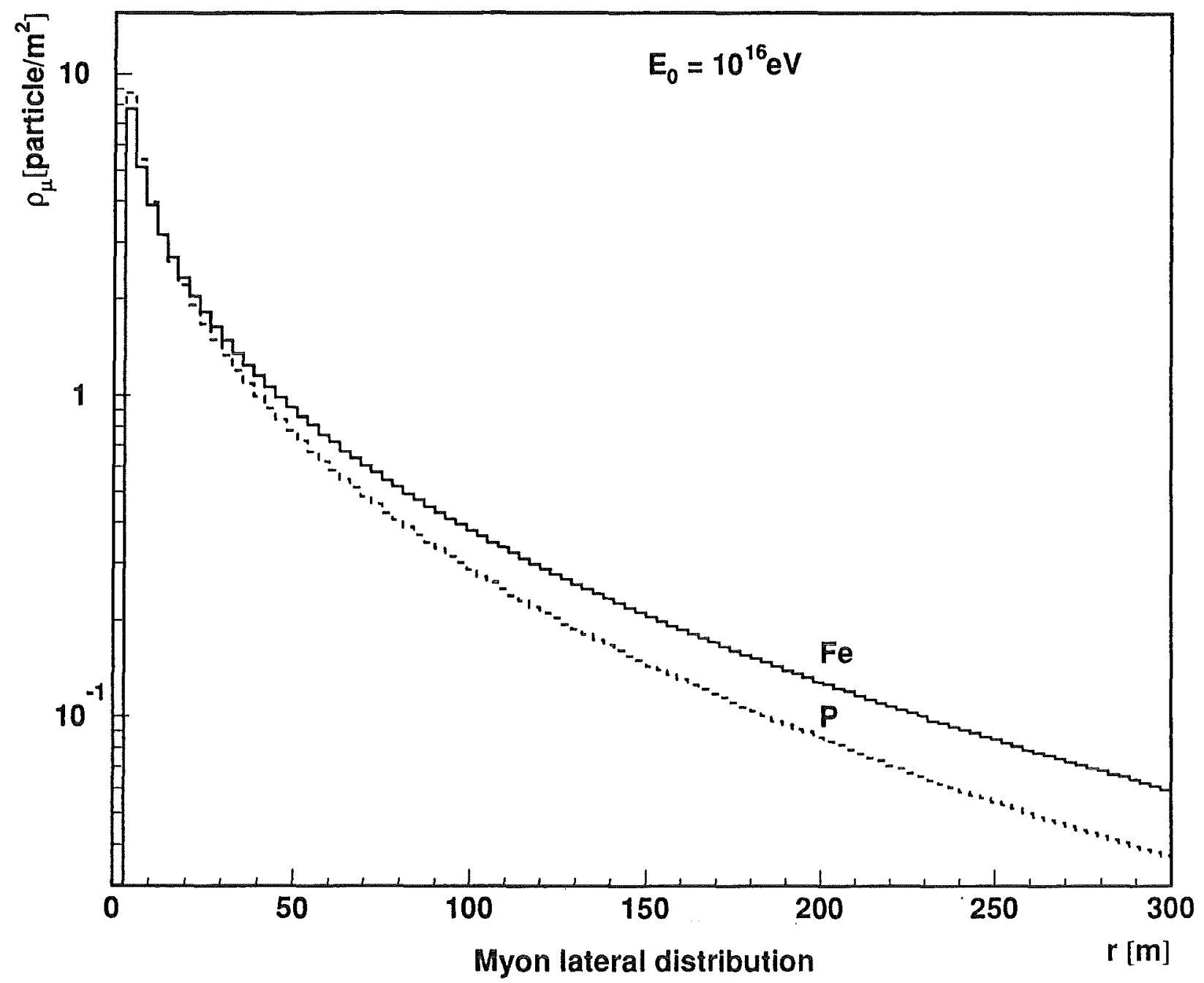

Fig. A8 